\title{
La fracturation et les bandes de déformation dans la région d'El Kohol (Atlas saharien central, Algérie): analyse fractale, lois d'échelles et modèle de réseaux de fractures discrètes
}

\author{
Fracturing and deformation bands in the El Kohol area \\ (Central Saharan Atlas, Algeria): fractal analysis, scaling \\ laws and discrete fracture network modelling
}

\author{
R.S. Zazoun ${ }^{1}$, A. Marok², L. Samar ${ }^{1}$, M. Benadla², H. Mezlah ${ }^{1}$ \\ 1 Sonatrach-Division Technologies et Développement, Avenue du $1^{\text {er }}$ Novembre, 35000 Boumerdès, Algérie. \\ Email: redasamy@hotmail.com \\ 2 Département of Earth and Univers Sciences, University of Tlemcen B.P. 119, Tlemcen, Algérie
}

\section{RÉSUMÉ}

Ce travail porte sur l'étude de la fracturation naturelle et les bandes de déformation dans la structure plicative d'El Kohol, du le Djebel Amour, dans l'Atlas saharien central. Les observations et les mesures ont été effectuées à travers deux stations sur le flanc court ou avant de la structure, et deux stations sur le flanc long ou arrière. L'étude a montré l'existence de cinq familles de fractures et de trois familles de bandes de déformation. Les modèles de distribution des espacements et des longueurs des différentes familles de fractures obéit à une loi de type puissance. L'analyse mécanostratigraphique montre une subdivision des formations étudiées en douze unités mécaniques. Les bandes de déformation montrent une augmentation de leurs nombres, ainsi qu'une réduction de leurs espacements à l'approche des accidents majeurs. Les analyses fractales effectuées sur les failles, les fractures et sur les bandes de déformation montrent un caractère fractal de dimension 2. La comparaison des paramètres de densité et d'intensité (Pxy) obtenus à partir de la modélisation du réseau de fractures discrètes (DFN) avec ceux calculés sur le terrain montrent de bons coefficients de corrélation. Le modèle établi est discuté à la lumière des phases de déformation reconnues dans la région.

Mots-clefs: Atlas Saharien; El Kohol; Fracturation; Réseau; Analyse fractale; Mécanostratigraphie; Modélisation stochastique.

\section{ABSTRACT}

The aim of this paper is focused on the study of natural fractures and deformation bands in El Kohol structure, located in the Djebel Amour in the Central Saharan Atlas, Algeria. The field observations and measurements were performed through two localities on the forelimb and two others on the backlimb of the structure.

Recibido el 7 de enero de 2015 / Aceptado el 22 de septiembre de 2015 / Publicado online el 06 de noviembre de 2015

Citation / Cómo citar este artículo: R.S. Zazoun, et al. (2015). La fracturation et les bandes de déformation dans la région d'El Kohol (Atlas saharien central, Algérie): analyse fractale, lois d'échelles et modèle de réseaux de fractures discrètes. Estudios Geológicos 71(2): e039. http://dx.doi.org/10.3989/egeol.42011.359.

Copyright: () 2015 CSIC. This is an open-access article distributed under the terms of the Creative Commons Attribution-Non Commercial (by-nc) Spain 3.0 License. 
The outcrop study has shown the existence of five fracture sets and three deformation bands sets. The spacing and length distribution models of the different fractures sets obey to a power law. The mechanical layer thickness analysis for the whole formations shows the existence of twelve mechanical units with a stratabound control. The deformation bands show an increasing in their numbers, and a decreasing in their spacing when they approach the major faults. The fractal analysis of faults and fractures, as well as the deformation bands show a fractal character of $2 \mathrm{D}$ dimension. A good correlation coefficients is obtained from the comparison between the density and the intensity parameters (Pxy) calculated from the discrete fracture network (DFN) modelling, and those from the outcrops. The model developed is discussed related to deformation events recognized in the area.

Keywords: Saharan Atlas; El Kohol; Fracturation; Network; Fractal analysis; Mecanostratigraphy; Stochastic modeling.

\section{Introduction}

La définition de la géométrie des réseaux de fractures (GRF) constitue un élément indispensable afin de modéliser les transferts de fluide, tels que les hydrocarbures dans les réservoirs naturellement fracturés (RNF). Observées de l'échelle microscopique à l'échelle continentale, les fractures sont des discontinuités mécaniques non-sédimentaires au sein d'une roche (Pollard \& Segall, 1987; National Research Council, 1996; Nelson, 2001; Macé, 2008). La description statique des fractures nécessite une hiérarchisation de concepts à savoir: du plus élémentaire (la fracture individuelle), à des intermédiaires qui sont la famille puis le système de fractures, et jusqu'a celui plus global de réseau (Macé, 2008). Les fractures d'un même système sont la conséquence de l'application d'un champ de contrainte commun (National Research Council, 1996). Par ailleurs, les caractéristiques de fracturation peuvent être modifiées localement dans le cas de fractures tectoniques liées à une faille majeure ou un pli (Peacock, 2001). Depuis les études de Mandelbrot (1975) sur les concepts de la géométrie fractale, de nombreux auteurs ont utilisé la technique de l'analyse fractale dans une tentative pour caractériser la géométrie à deux dimensions des réseaux de fractures (Allègre et al., 1982; Turcotte, 1986; Chilès, 1988; Turcotte, 1992; Gillespie et al., 1993; Bodin \& Razack, 1999; Bonnet et al., 2001; Zazoun, 2008; Kruhl, 2013). Selon Mandelbrot (1975), le terme d'auto-similarité est utilisé pour les objets où des parties de l'objet ressemblent à l'ensemble. La loi de puissance est souvent désignée comme représentative de schéma auto-similaires dont l'exposant représente les dimensions fractales (Odling et al., 1999).
Notre travail porte sur l'étude de la fracturation naturelle et les bandes de déformation dans la structure plicative d'El Kohol, située au sud-est de Brézina, fait partie de la grande barrière orographique du Djebel Amour (Atlas saharien central) (Fig. 1A et 1B). De direction E-O, en bordure de l'Accident Sud Atlasique, la structure d'El Kohol est un anticlinal étroit avec un flanc nord (long) régulier à pendage ne dépassant pas les $50^{\circ}$, alors que le flanc sud (court) montre des pendages fortement redressés (Bettahar et al., 1996). cette structure à fait l'objet de nombreuses études pluridisciplinaires et son histoire géologique et sa cinématique sont relativement bien connues grâce à la qualité des affleurements constitués essentiellement de dépôts gréseux, argilogypseux ou encore carbonatés (Bettahar et al., 1996; Habani \& Haddoum, 2006; Guemache et al., 2011; Rerbal, 2008) permettant l'étude des modes de déformation actifs simultanément dans des lithologies différentes ayant subi la même histoire de déformation. Hormis, ces études, aucun travail n'a été entrepris dans le domaine de la fracturation naturelle. Ainsi, l'objectif de cette note est: (1) donner en revue la terminologie et les mécanismes de la fracturation; (2) hiérarchiser la fracturation observée à l'affleurement en familles de fractures, puis en système de fractures, et jusqu'a celui du réseau; (3) analyser la distribution de la longueur, de l'orientation et de l'espacement des fractures; (4) appliquer le concept de la géométrie fractale et tester si le modèle répond à une loi de type puissance; (5) établir une image réaliste du réseau de fractures compatible avec les modèles mathématiques et puis, (6) discuter des mécanismes et de la chronologie des systèmes de fractures. La génération stochastique d'un modèle de réseaux de fractures discrètes (DFN) sera également discutée ici. 


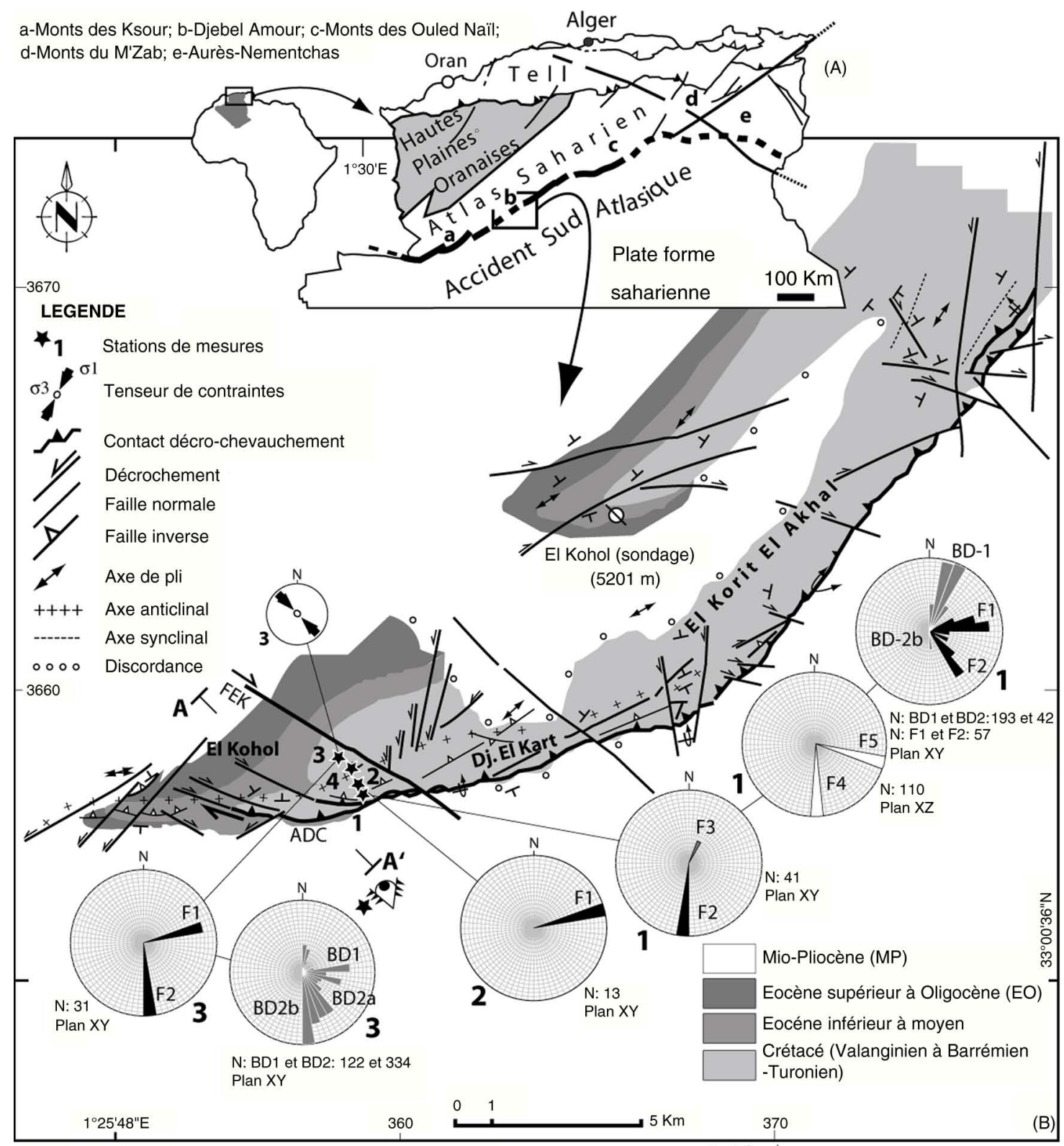

F1, F2, F3, F4 et F5: Familles de fractures BD1 et BD2: Bandes de déformation FEK: Faille d'El Kohol

ADC: Accident décro-chevauchant d'El Kohol $A^{\prime}$ : Coupe géologique (Bracène et al., 1999)

1, 2, 3 et 4: Stations de mesures; CT: Cénomano.-Turonien

Site d'observation panoramique (figure 7 , station 4)

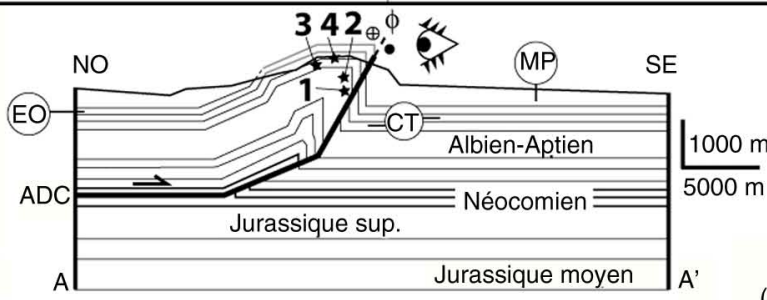

Fig. 1.-Localisation et schéma structural du secteur d'étude. (A) Localisation géographique et géologique de la région d'El Kohol. (B) Distribution de la fracturation et des bandes de déformation au niveau de la région d'El Kohol (Schéma structural d'après Bettahar et al., 1996; Habani \& Haddoum, 2006). (Reconstitution des tenseurs de contrainte pour la station 4 à l'aide du logiciel FaultKin 6.1.0 (Marett \& Allmendinger,1990; Allmendinger et al., 2012; Allmendinger, 2013). (C) Modélisation géologique de la structure d'El Kohol (D'après Bracène et al., 1999). 


\section{Cadre géologique}

\section{Structural}

Le domaine atlasique au sens large (Rerbal, 2008) correspond à trois grands ensembles morphologiques limités au Nord comme au Sud par deux accidents majeurs. Il s'agit de l'Ouest vers l'Est: l'Atlas saharien occidental (Monts des Ksour), l'Atlas saharien central (Djebel Amour) et l'Atlas saharien oriental (Ouled Naïl, Aurès-Nementchas). Limité au Nord-Ouest par les Hautes Plaines Oranaises (Marok, 1996), au Sud par la plate-forme saharienne, à l'Est par les Monts d'Ouled Naïl et à l'Ouest par la terminaison orientale des Monts des Ksour. (Fig. 1A). L'Atlas saharien central, objet de cette présente étude a été le siège d'une tectonique hercynienne polyphasée qui a influencée la sédimentation et guidée les phases de déformation durant le Méso-Cénozoïque (Bettahar et al., 1996). A l'aide de l'interprétation des sections sismiques dans la région d'El Kohol (Fig. 1B), Habani \& Haddoum (2006), évoquèrent l'existence d'une tectonique transcurrente qui aurait engendré un système de rampes et paliers avec des surfaces de décollement qui se situeraient au Jurassique et au Néocomien-Barrémien. Pour la même région, Guemache et al. (2011), propose un modèle, dans lequel le pli d'El Kohol correspondrait à un front de chevauchement à vergence sud, impliquant des plis passifs sur rampe qui se relaient plus en avant à des plis de propagation (Fig. 1C). Selon ces auteurs, le principal niveau de décollement tectonique se situerait au niveau du Crétacé inférieur au sudouest et du Jurassique au nord-est, à l'arrière des plis susmentionnés. Bettahar et al. (1996) mettent en évidence dans la région d'El Kohol trois phases majeurs de déformation: (1) la première transcurrente sénestre d'âge Crétacé supérieur, responsable d'un raccourcissement NE-SO. (2) la seconde phase transcurrente dextre traduisant un raccourcissement NO-SE d'âge Eocène supérieur. (3) la troisième compressive, compatible avec un raccourcissement N-S d'âge Mio-Pliocène. Une quatrième phase non argumentée, d'âge probable Plio-Quaternaire réactivant les failles antérieures.

\section{Lithostratigraphie}

La région d'étude se caractérise par les dépôts d'une épaisse série sédimentaire couvrant l'intervalle stratigraphique Mésozoïque-Cénozoïque. La nomenclature des différentes formations étudiées ici est adaptée de celle de Rerbal (2008). Ainsi, on distingue dans l'ordre ascendant la succession lithostratigraphique suivante:

a) La Formation d'El Rhelida: incomplète dans la région d'étude (18 $\mathrm{m}$ d'épaisseurs), cette formation est attribuée à l'Albien supérieur (Vraconien). Elle est constituée essentiellement d'une série argilo-gréseuse admettant quelques niveaux carbonatés (calcaires et dolomies). Stratigraphiquement, la série repose sur les dépôts deltaïques de la formation de Tiout d'âge Néocomien-Albien (Rerbal, 2008).

b) La Formation de M'daouer: épaisse de 99,50 mètres et d'âge Cénomanien inférieur, cette formation est représentée par une alternance de marnes à gypses et de bancs calcaires parfois dolomitiques.

c) La Formation de Rhoundjaïa: formant une corniche géomorphologiquement repérable, la Formation de Rhoundjaïa est épaisse de 57,5 mètres et d'âge Cénomanien supérieurTuronien inférieur. Elle est constituée de dépôts carbonatés formés de calcaires et de calcaires dolomitiques admettant parfois des inter-bancs marneux très riches en microfaune (foraminifères et ostracodes). Notons également la présence de la macrofaune (ammonites, bivalves, gastéropodes, échinodermes).

Enfin les formations rapportées au Paléogène et au Néogène sont représentés par des dépôts détritiques essentiellement continentaux ne dépassant pas 1000 m. (Guillemot \& Estorges, 1981; Rerbal, 2008).

\section{Acquisition et traitement des données}

Les observations et les mesures des fractures et des bandes de déformation ont été effectuées à travers 4 stations de mesures, 2 stations sur le flanc court ou avant (forelimb) de la structure ( 1 et 2 ) et 2 stations sur le flanc long ou arrière (backlimb) (3 et 4) (Fig. 1B; Tableau 1). La méthodologie d'approche consiste à:

- A analyser la fractalité du réseau de failles à partir de la carte géologique (Fig. 1B), ainsi que la géométrie fractale en 2 dimensions des mesures de réseaux de fracturation et des bandes 
Tableau 1.-Localisation géographique des stations de mesures et d'observations dans la région d'El Kohol. De gauche vers la droite: $N^{\circ}$ de la station; coordonnées GPS; nom et âge de la formation

\begin{tabular}{|c|c|c|c|c|c|c|}
\hline \multirow{2}{*}{$\begin{array}{c}\mathrm{N}^{\circ} \\
\text { Station }\end{array}$} & \multicolumn{3}{|c|}{ Coordonnées GPS } & \multicolumn{2}{|c|}{ Géologie } & \multirow[b]{2}{*}{ Remarques } \\
\hline & $\mathrm{x}$ & $\mathrm{Y}$ & $\mathrm{Z}(\mathrm{m})$ & Formations & Age & \\
\hline 1 & $1^{\circ} 30,0177^{\prime} \mathrm{E}$ & $33^{\circ} 3,770^{\prime} \mathrm{N}$ & 907 & El Rhelida & Albien sup. (Vraconien) & Flanc court ou avant \\
\hline 2 & $1^{\circ} 29,9325^{\prime} \mathrm{E}$ & $33^{\circ} 3,3189^{\prime} \mathrm{N}$ & 953 & El Rhelida & Albien sup. (Vraconien) & Flanc court ou avant \\
\hline 3 & $1^{\circ} 29,5610^{\prime} \mathrm{E}$ & $33^{\circ} 3,7461^{\prime} \mathrm{N}$ & 930 & M'daouer-Rhoundjaïa & Cénom. inf.-Turon. inf. & Flanc long ou arrière \\
\hline 4 & $1^{\circ} 30,0878^{\prime} \mathrm{E}$ & $33^{\circ} 3,2921^{\prime} \mathrm{N}$ & 920 & El Rhelida-M'daouer-Rhoundjaïa & Albien sup.-Turon. inf. & Flanc long ou arrière \\
\hline
\end{tabular}

de déformation relevées sur le terrain, afin de calculer la dimension fractale à l'aide du logiciel Fractalyse 2.3.2 développé par Frankhauser et al. (2005) selon la méthode "The Two Point Correlation Function Method" de Hentschel \& Procaccia (1983) et Grassberger \& Procaccia (1983). (Equation 1),

$$
C 2(r)=1 / N^{2} * N d(r)
$$

- Avec $N$ étant le nombre total de points et $N d$, le nombre de paires de points dont la distance est inférieure à $r$. Pour une population fractale de points, $C 2(r)$ est prévu avec une échelle où $(r)$ est égal à $(r)$ élevé à la puissance $D c$, où $D c$ représente la dimension de corrélation du système. Cette technique est basée sur la distribution spatiale des barycentres des traces de failles ou fractures définis comme les points médians (Davy et al., 1990; Sornette et al., 1993, Bour \& Davy, 1999).

- A lever des profils d'échantillonnage (scan line) perpendiculairement aux plans de fractures et des bandes de cisaillement d'une même famille (dans le plan XY) afin de déterminer les types de lois d'espacements (Fig. 2). Les mesures d'espacements ont été effectuées au mètre-ruban à manivelle d'une longueur de 20 mètres. Si les fractures ne sont pas perpendiculaires au profil d'échantillonnage on procède à une correction selon la loi de Terzaghi (1965) et Narr (1996) (Equation 2),

Espacement réel $=$ Espacement mesuré $* \mathrm{~W}$ avec $\mathrm{W}=\cos \left(90^{\circ}-\theta\left(^{\circ}\right)\right)$

$\theta$ étant l'angle en degré entre le profil d'échantillonnage et les fractures d'une même famille subparallèles. Le traitement des données de fracturation à l'affleurement ont été analysés à l'aide du logiciel Matter Cliff 1.0 développé

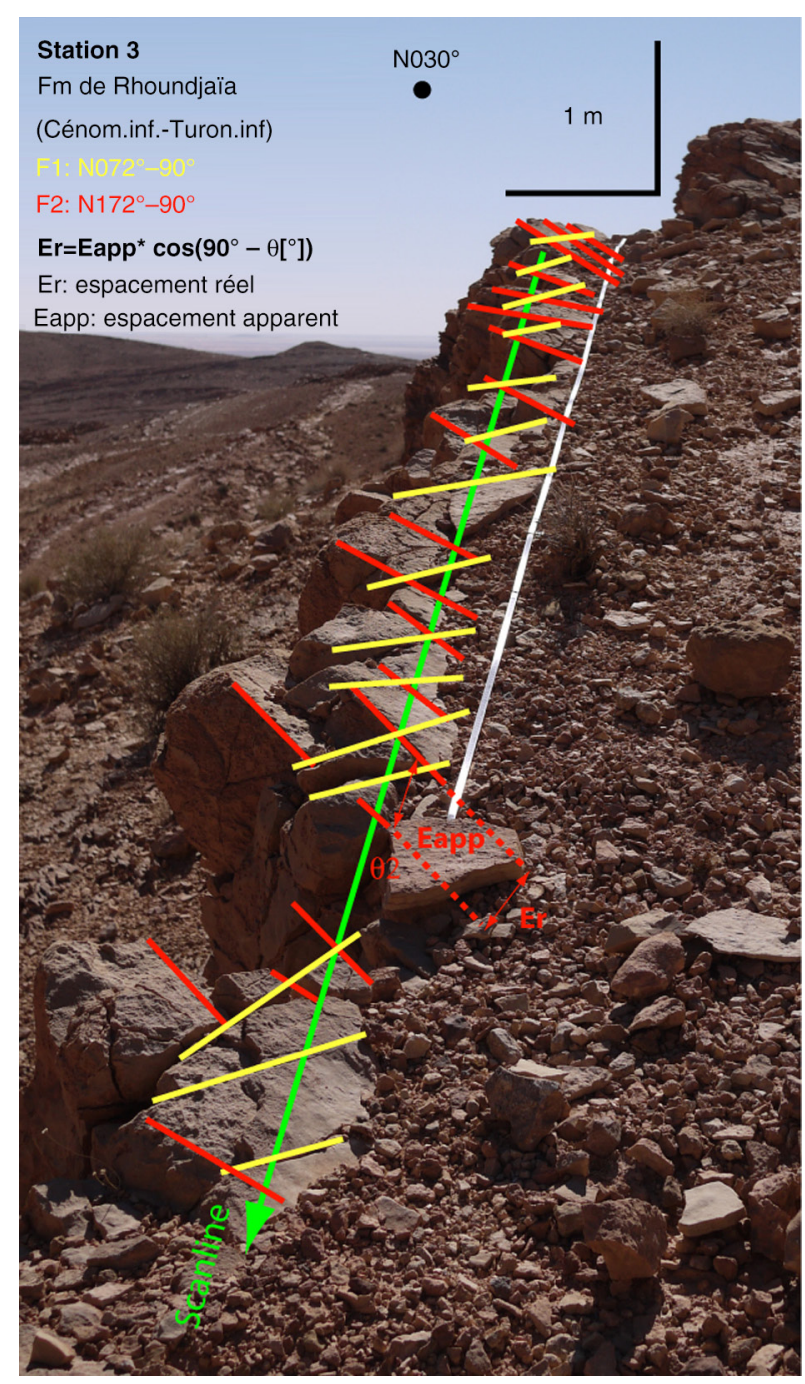

Fig. 2.-Exemple de profil d'échantillonnage tracé pour l'étude d'un réseau de fracturation (Station 3, Formation de Rhoudjaïa). 
par Deleze et al. (2005) pour l'étude statistique et la distribution des familles de discontinuités, et l'établissement des types de lois des espacements.

- A calculer les paramètres d'intensité et de densité (Pxy). A savoir, P0, P10, P20, P21 et P32 des fractures sur un profil d'échantillonnage selon le modèle de Dershowitz \& Herda (1992).

- A étudier la fracturation de chaque banc au sein de chaque formation (dans le plan XZ) afin de caractériser les unités mécaniques.
- A générer stochastiquement un modèle de réseaux de fractures discrètes (DFN) à l'aide du logiciel FracSim3D développé par Xu \& Dowd. (2010).

\section{Etude du réseau de failles}

\section{Analyse spatiale fractale du réseau $2 D$ de failles}

L'analyse fractale effectuée par la méthode "The Two Point Correlation Function Method" pour la carte de la région d'El Kohol (Fig. 3A) montre un

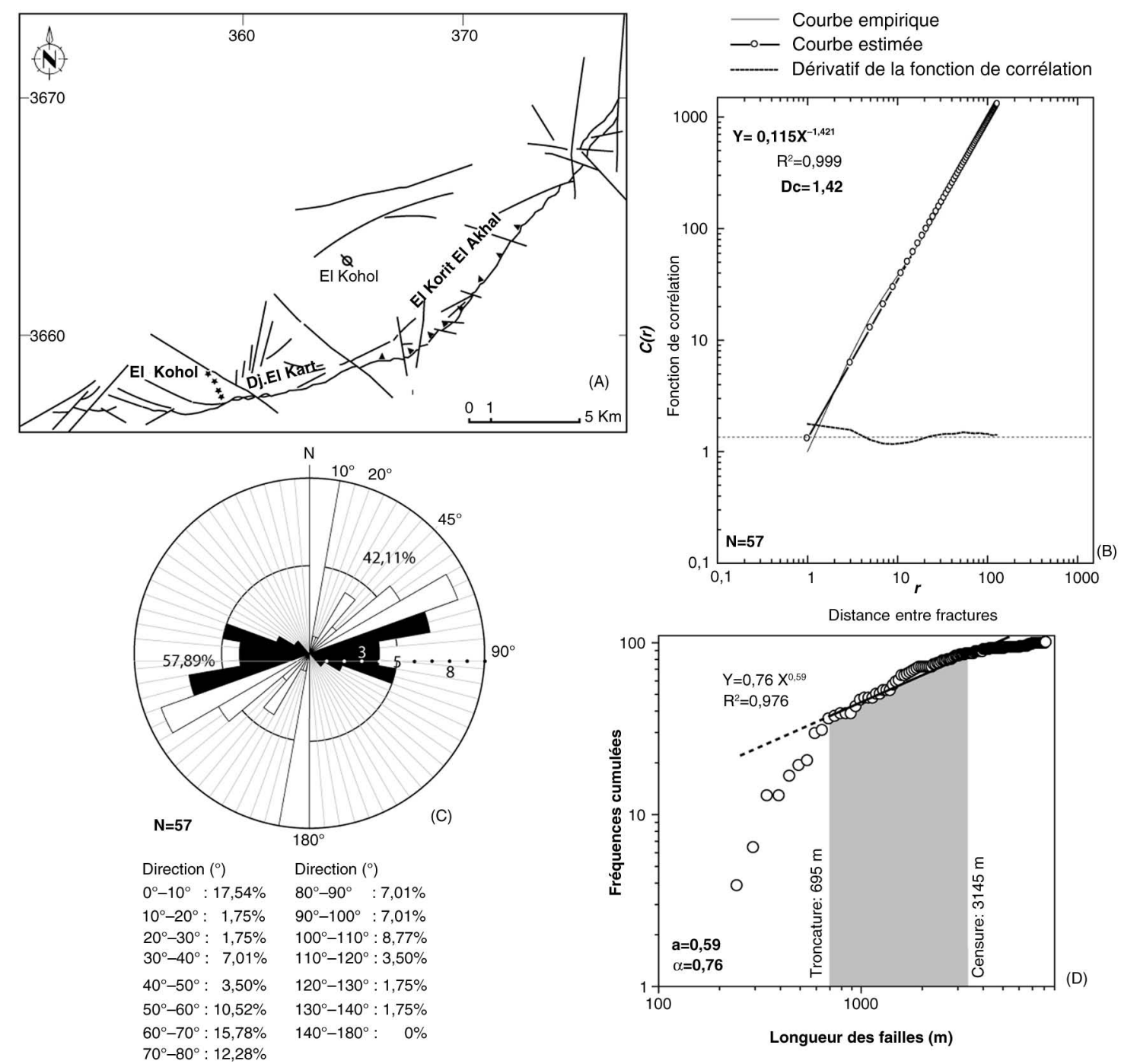

Fig. 3.-Analyse du réseau de failles dans la région d'El Kohol. (A) Carte de la répartition des failles. (B) Résultat de l'analyse fractale du réseau de failles par la méthode "The Two Point Correlation Function Method". La régression entre les couples de valeurs [Cr, $r$ ] peut être ajustée par une loi de puissance. Le réseau de failles peut être assimilé à un objet fractal de dimension $D c=1,42$. (C) Rosace d'orientation des failles dans la région d'El Kohol. (D) Diagramme bi-logarithmique de la distribution des fréquences cumulées des longueurs des failles. 
caractère fractal de dimension 2 pour la répartition des failles. En effet, la courbe estimée du graphe bi-logarithmique de la fonction de corrélation $[C(r)]$ en fonction de la distance entre fractures $(r)$ peut être ajustée par une loi de puissance (Fig. 3B). La dimension fractale $(D c)$ obtenue est de 1,42; donc comprise entre 1 et 2 , avec un coefficient de corrélation $\left(\mathrm{R}^{2}\right)$ de 0,99 . Cette valeur $D c$ calculée est cohérente avec les résultats publiés dans la littérature sur les études en 2D des réseaux de fracturation (Bonnet et al., 2001).

\section{Analyse de l'orientation des failles}

L'interprétation de la rosace des orientations des failles dans la région d'El Kohol (Fig. 3C) a permis de distinguer une famille N-S (dextre), NO-SE (dextre) et NE-SO (senestre) avec des proportions respectivement de l'ordre de $17,54 \%, 14,02 \%$ et $10,51 \%$.

\section{Distribution des longueurs de failles}

A partir du fichier des données de la carte numérisée, la longueur des failles à été calculée puis analysée. Les longueurs des fractures sont comprises dans l'intervalle [220,34 m: 8161,50 m] et s'étendent sur plusieurs ordres de grandeurs. Le diagramme log-log des données de fréquences cumulées des longueurs (classes de longueur $=50 \mathrm{~m}$ ) est représenté sur la figure 3D. Seule une partie de la courbe entre $695 \mathrm{~m}$ et $3145 \mathrm{~m}$ à un comportement linéaire et peut être ajustée par une loi de puissance présentant une droite de pente (a) égal à 0,59 avec un coefficient de détermination de 0,97. La loi de puissance (Fig. 3D) déduite à partir de la distribution des fréquences, ne rend pas compte des valeurs inférieures à $695 \mathrm{~m}$ et supérieures à $3145 \mathrm{~m}$. Ces biais sont causés par les effets de taille des failles. Les grandes failles présentent une probabilité plus élevée d'être échantillonnées que les plus petites et les failles plus courtes qu'une longueur donnée ne sont pas échantillonnées. D'après Odling et al. (1999), la distribution des longueurs de failles selon une loi de puissance présentent des seuils inférieurs (troncature) et des seuils supérieurs (censure), au deçà et au-delà desquels la loi de puissance n'est pas valide. La distribution en loi de puissance se situe entre $695 \mathrm{~m}$ et $3145 \mathrm{~m}$ et couvrent seulement 2 ordres d'échelle après le retrait des artefacts de censure et de troncature.

\section{Étude du réseau de fractures}

Dans notre approche, nous avons considéré sur un profil d'échantillonnage l'espacement entre fractures appartenant à une même famille par stations de mesures (Fig. 1B). Selon Bodin \& Razack (1999), les travaux sur les lois de distribution des espacements entre les fractures d'un réseau remontent à plusieurs dizaines d'années (Priest \& Hudson, 1976), et demeurent encore sujet de controverse (Dershowitz $\&$ Einstein, 1988). Dans cette optique, des différentes lois de distribution ont été ajustées aux données d'espacement: loi log-normale, négative exponentielle, loi normale et loi de puissance (Bonnet et al., 2001; Peacock \& Mann, 2005; Zazoun, 2008; Kruhl, 2013).

\section{Station 1}

Situé sur le flanc court de la formation d'El Rhelida, l'affleurement (Fig. 1B) se distingue par l'existence de deux familles de fractures. Une famille F2 $\left(\mathrm{N} 185^{\circ}-90^{\circ}\right)$ et une famille F3 (N030 $\left.-24^{\circ} \mathrm{SE}\right)$ ouvertes pour la plus part, portées par une stratification S0: N080 $-75^{\circ} \mathrm{S}$. L'analyse des espacements entre fractures, le long du profil d'échantillonnage montre une distribution de type log-normale pour les deux familles en accord avec un modèle dit aléatoire (Fig. 4A et 4B). Les valeurs moyennes de l'espacement sont respectivement de $16,83 \mathrm{~cm}$ et $5,06 \mathrm{~cm}$, pour les familles F2 et F3. Le coefficient de variation reste inférieur à $1(0,83$ pour $F 2$ et 0,95 pour $F 3)$, ce qui permet de souligner un contrôle de la fracturation par l'épaisseur des bancs (stratabound) (Odling et al., 1999). Par ailleurs, le rapport de la médiane sur la moyenne proche de la valeur 1 pour les deux familles suggère une géométrie de réseau saturé. La distribution des fréquences cumulées des espacements entre fractures montre pour la famille F2 une loi de puissance entre 233,5 cm et 473,5 cm (Fig. 4C), caractérisée par une droite de pente $-2,51\left(\mathrm{R}^{2}=0,98\right)$. A l'instar de la famille F2, la famille F3 montre une loi de puissance entre $3,5 \mathrm{~cm}$ et $45,5 \mathrm{~cm}$ avec une valeur de pente $-0.33\left(\mathrm{R}^{2}=0,91\right)$ (Tableau 2$)$.

\section{Station 2}

Localisé au sud du flanc court dans la formation d'El Rhelida, L'affleurement de la station 2 (Fig. 1B) montre une famille de fracture $\mathrm{F} 1$ (N075 $\left.-84^{\circ} \mathrm{NNO}\right)$ 


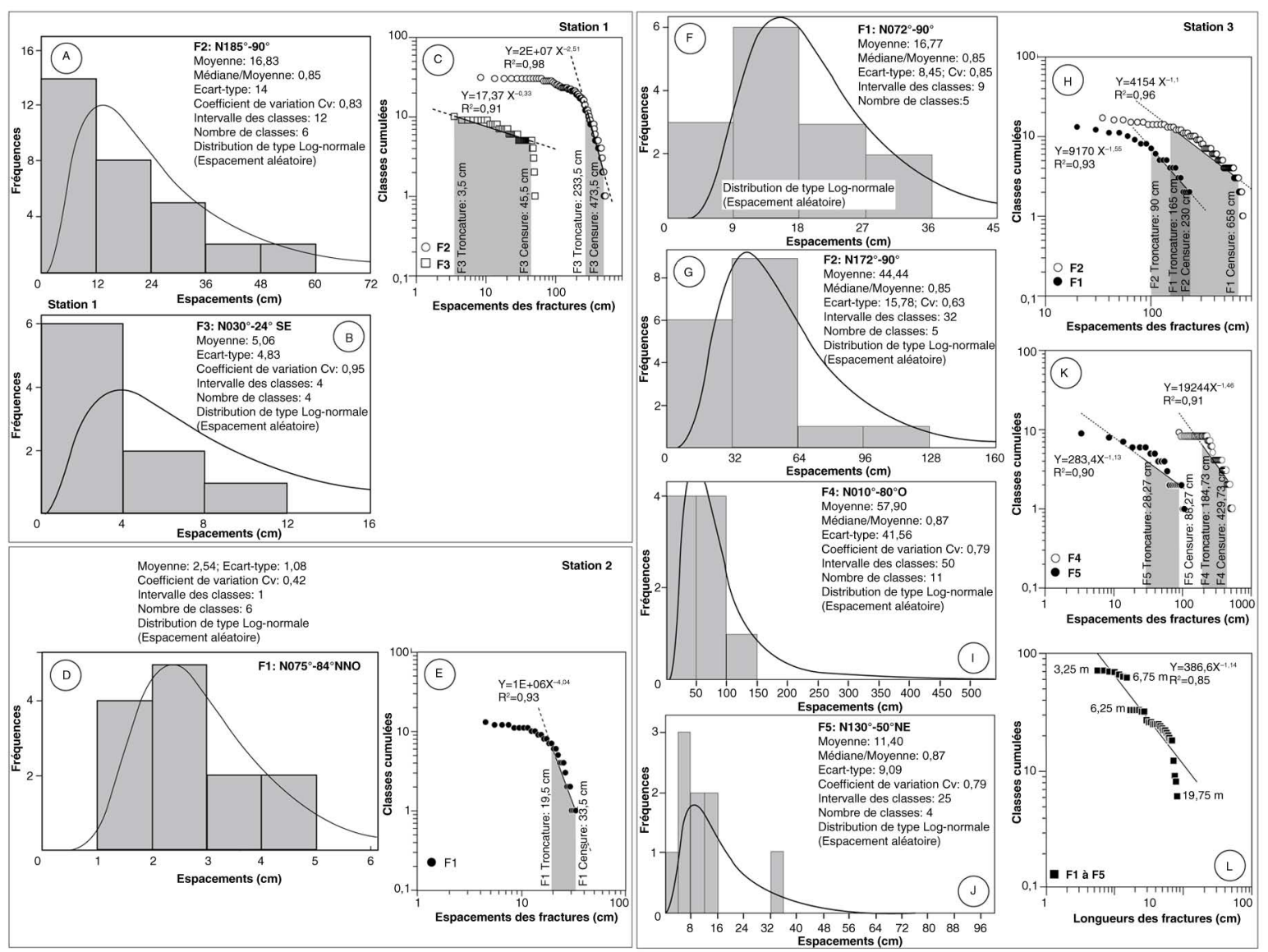

Fig. 4.-Profil d'échantillonnage aux niveaux des stations 1, 2 et 3. (A) Histogramme des fréquences en fonction des espacements entre fractures et loi de distribution (Famille de fracture F2). (B) Histogramme des fréquences en fonction des espacements entre fractures et loi de distribution (Famille de fracture F3). (C) Diagramme bi-logarithmique de la distribution des fréquences cumulées des espacements entre fractures (Familles de fracture F2 et F3). (D) Histogramme des fréquences en fonction des espacements entre fractures et loi de distribution (Famille de fracture F1). (E) Diagramme bi-logarithmique de la distribution des fréquences cumulées des espacements entre fractures (Famille de fracture F1). (F) Histogramme des fréquences en fonction des espacements entre fractures et loi de distribution (Famille de fracture F1). (G) Histogramme des fréquences en fonction des espacements entre fractures et loi de distribution (Famille de fracture F2). (H) Diagramme bi-logarithmique de la distribution des fréquences cumulées des espacements entre fractures (Familles de fractures F1 et F2). (I) Histogramme des fréquences en fonction des espacements entre fractures et loi de distribution (Famille de fracture F4). (J) Histogramme des fréquences en fonction des espacements entre fractures et loi de distribution (Famille de fracture F5). (K) Diagramme bi-logarithmique de la distribution des fréquences cumulées des espacements entre fractures (Familles de fractures F4 et F5). (L) Diagramme bi-logarithmique de la distribution des fréquences cumulées des longueurs des fractures (plan XY; Familles de fractures F1 à F5).

ouverte, portée par une stratification $\mathrm{S} 0$ : $\mathrm{N} 080^{\circ}-75^{\circ} \mathrm{SSE}$. L'analyse des espacements entre fractures, le long du profil d'échantillonnage montre une distribution de type log-normale en accord avec un modèle dit aléatoire (Fig. 4D). La valeur moyenne de l'espacement est de $2,54 \mathrm{~cm}$. Le coefficient de variation reste inférieur à 1 (il est de 0,42 ), ce qui permet de souligner le contrôle de la fracturation par l'épaisseur des bancs. Le rapport de la médiane sur la moyenne proche de la valeur 1 suggérant une géométrie de réseau saturé. La distribution des fréquences cumulées des espacements entre fractures montre pour la famille F1, une loi de puissance entre 19,5 cm et 33,5 cm (Fig. 4E), caractérisée par une droite de pente $-4,04\left(\mathrm{R}^{2}=0,93\right)$ (Tableau 2).

\section{Station 3}

A la différence des affleurements précités, cet affleurement se situe sur le flanc long de la structure et il intéresse les formations de M'daouer et 


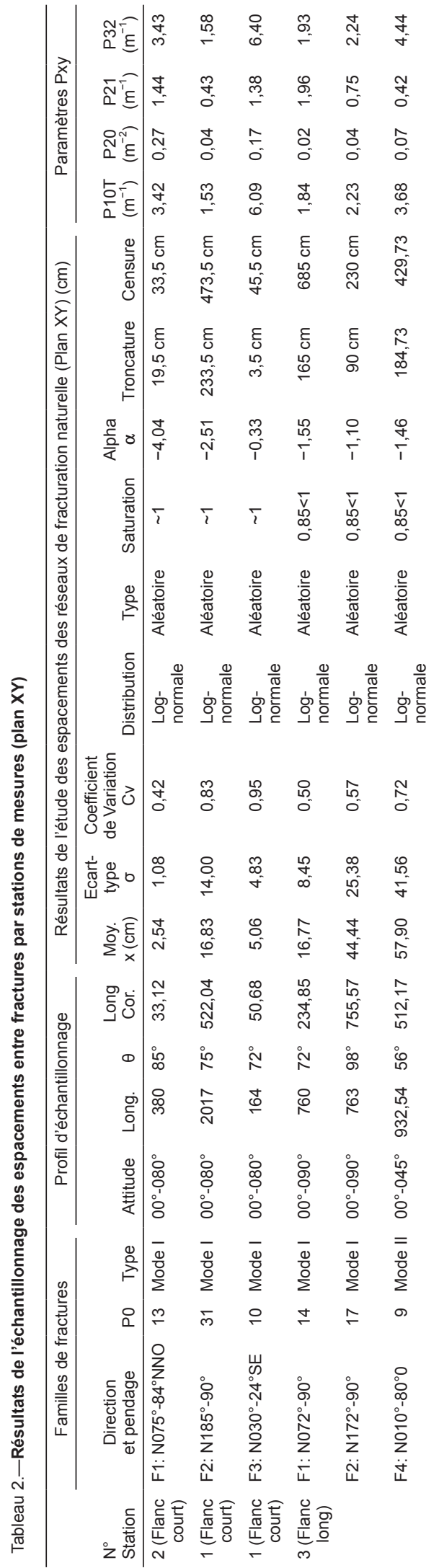

Rhoundjaïa (Fig. 2). Il montre l'existence de quatre familles de fractures:

Une famille $\mathrm{F} 1\left(\mathrm{~N} 072^{\circ}-90^{\circ}\right)$, une famille $\mathrm{F} 2$ $\left(\mathrm{N} 172^{\circ}-90^{\circ}\right)$, une famille $\mathrm{F} 4\left(\mathrm{~N} 010^{\circ}-80^{\circ} \mathrm{O}\right)$ et une famille $\mathrm{F} 5\left(\mathrm{~N} 130^{\circ}-50 \mathrm{NE}^{\circ}\right)$ portées par une stratification $\mathrm{S} 0$ : $\mathrm{N} 030^{\circ}-18^{\circ} \mathrm{NO}$.

L'analyse des espacements entre fractures, le long du profil d'échantillonnage montre une distribution de type log-normale pour les familles F1, F2, F4 et F5 en accord avec un modèle dit aléatoire (Fig. 4F, 4G, 4I et 4J). Les valeurs moyennes de l'espacement sont respectivement de $16,77 \mathrm{~cm}, 44,44 \mathrm{~cm}$, $57,90 \mathrm{~cm}$ et $11,40 \mathrm{~cm}$ pour les familles $\mathrm{F} 1, \mathrm{~F} 2, \mathrm{~F} 4$ et $\mathrm{F} 5$. Le coefficient de variation reste inférieur à 1 $(0,50$ pour $F 1,0,63$ pour $F 2,0,72$ pour $F 4$ et 0,79 pour F5), ce qui permet de souligner un contrôle de la fracturation par l'épaisseur des bancs. Le rapport de la médiane sur la moyenne proche de la valeur 1 pour les cinq familles suggère une géométrie de réseau saturé. La distribution des fréquences cumulées des espacements entre fractures montre pour la famille $\mathrm{F} 1$ une loi de puissance entre $165 \mathrm{~cm}$ et $685 \mathrm{~cm}$ (Fig. 4H), caractérisée par une droite de pente $-1,55\left(\mathrm{R}^{2}=0,93\right)$. La famille $\mathrm{F} 2$ montre une loi de puissance entre $90 \mathrm{~cm}$ et $230 \mathrm{~cm}$ avec une valeur de pente $-1.1\left(\mathrm{R}^{2}=0,96\right)$. Par contre, la distribution des fréquences cumulées des espacements entre fractures montre pour la famille F4 une loi de puissance entre $184,73 \mathrm{~cm}$ et $429,73 \mathrm{~cm}$, caractérisée par une droite de pente $-1,46\left(\mathrm{R}^{2}=0,91\right)$ (Fig. $\left.4 \mathrm{~K}\right)$. La famille F5 montre une loi de puissance entre $28,27 \mathrm{~cm}$ et $88,27 \mathrm{~cm}$ avec une valeur de pente $-1,13\left(\mathrm{R}^{2}=0,90\right)$ (Tableau 2).

La distribution des fréquences cumulées des longueurs des fractures à l'affleurement (plan XY) montre pour les fractures toutes familles confondues une loi de puissance entre 3,25 m et 19,75 m caractérisée par une droite de pente $-1,14\left(\mathrm{R}^{2}=0,85\right)($ Fig. $4 \mathrm{~L})$.

\section{Distributions des paramètres Pxy}

Nous avons calculées la distribution des paramètres $\mathrm{P} 0, \mathrm{P} 10, \mathrm{P} 20, \mathrm{P} 21$ et $\mathrm{P} 32$ pour l'ensemble des familles de fractures dans le plan XY. Les résultats sont présentés dans le Tableau 2.

P0 étant le nombre total de fractures pour une même famille,

avec $\mathrm{P} 0=\mathrm{N}$ 
P10 étant la densité linéique ou le nombre de fractures observées pour une même famille interceptées sur un profil d'échantillonnage de longueur L. L'inverse de P10 représente l'espacement moyen des fractures d'une même famille (Ortega et al., 2006).

avec $\mathrm{P} 10=\mathrm{N} / \mathrm{L}$

P20 est le nombre de fractures d'une même famille par unité de surface $\left(\mathrm{m}^{2}\right)$,

avec $\mathrm{P} 20=\mathrm{N} / \mathrm{m}^{2}$

P21 représente la longueur totale des fractures d'une même famille (mètre) dans une zone spécifiée $\left(\mathrm{m}^{2}\right)$,

avec $\mathrm{P} 21=\mathrm{L}$ totale par $\mathrm{m}^{2}$

P32 correspond à la densité tridimensionnelle de fracturation et elle est égale à la somme des surfaces des fractures par unité de volume.

avec $\mathrm{P} 32=\mathrm{S}\left(\mathrm{m}^{2}\right) / \mathrm{m}^{3}$

dans le cas des fractures parallèles

$\mathrm{P} 32=\csc (\theta) * \mathrm{P} 10$

Le flanc court ou avant de la structure montre un P0 égal à 54 (familles de fractures F1, F2 et F3), alors que le flanc long ou arrière, le $\mathrm{P} 0$ est égal à 49 (familles de fractures F1, F2, F4 et F5). Au niveau $\mathrm{du}$ flanc court, nous avons remarqué que la valeur importante de P10 pour la famille F3 (P10=6,09), est liée probablement au faible espacement moyen des fractures $(5,06 \mathrm{~cm})$ à la proximité du plan axial $\mathrm{du}$ pli. Pour le flanc long ou arrière la forte valeur de P10 est observée pour la famille F4 $(\mathrm{P} 10=3,68)$ et F5 $(\mathrm{P} 10=8,76)$. Cette forte valeur constitue une réponse à l'influence de la longueur de la fracture sur la distribution de P10 (Bisdom, 2011). La valeur du P20 est généralement inférieure au P10. Ceci est le cas pour toutes les familles observées aux niveaux des stations et cela signifie que les fractures courtes sont prises en considération et elles sont mesurées. Toutefois, la famille F2 sur les deux flancs présente une valeur de P20 identique et égale à 0,04 . La famille de fracture F1 montre des valeurs de P20 différentes pour les stations 2 et 3 . La forte valeur $(0,27)$ de cette dernière est observée sur le flanc court et elle est liée également au faible espacement moyen des fractures $(2,54 \mathrm{~cm})$ et de la proximité du plan axial du pli. Quant aux familles F4 et F5, elles présentent la même valeur de P20, égale à 0,07 . Sans exceptions, la distribution de $\mathrm{P} 21$ est très similaire à la distribution du P10. Sa distribution a l'avantage sur le P20 qu'elle ne dépend pas uniquement du nombre de fractures, mais aussi sur la longueur des fractures (Bisdom, 2011). L'utilisation du paramètre P21 en 2D calculé comme une fonction de la distribution des longueurs de fractures peut être utilisé pour prédire la distribution de l'espacement d'une famille de fractures (Fig. 5A).

Le graphe bi-logarithmique du paramètre $\mathrm{P} 32$ en fonction du paramètre P10 d'une part et le graphe bi-logarithmique du paramètre P32 en fonction du paramètre P20 d'autre part montrent une relation proportionnelle (Fig. 5B et 5C).

Le flanc long ou arrière de la structure présente un réseau ou les fractures sont plus longues et moins denses. A l'inverse, le flanc court ou avant présente les fractures les moins longues et les plus denses. Il est à noter que la connectivité d'un réseau de fracturation peut être estimée de manière qualitative en regardant la densité de fractures du P21. Lorsque le P21 est élevé, cela signifie que le potentiel d'écoulement est plus favorable du fait des grandes longueurs de fractures et lorsque les paramètres de densité P10 et $\mathrm{P} 20$ sont presque identiques, il est attendu à ce que le réseau de fractures présente des caractéristiques d'écoulement de fluide (connectivité et capacité de stockage) similaires (Bekkers, 2013). Dans cette analyse, nous distinguons que la famille de fracture F1 présente le plus grand potentiel d'écoulement $(1,44 \leq \mathrm{P} 21 \leq 1,96)$ sur les deux flancs de la structure et ceci malgré que le réseau présente des caractéristiques d'écoulement de fluide non similaires (P10 différent de P20) (Tableau 2 et Fig. 5D).

\section{Station 4: Mécanostratigraphie des formations d'El Rhelida, M'daouer et Rhoundjaïa}

L'unité mécanique (Gross, 1993; Laubach et al., 2009) correspond à une ou à un groupe de couches qui affichent des motifs de ruptures similaires, selon des attributs géométriques et des propriétés physiques de la roche hôte (Lavenu et al., 2013). Nous avons 


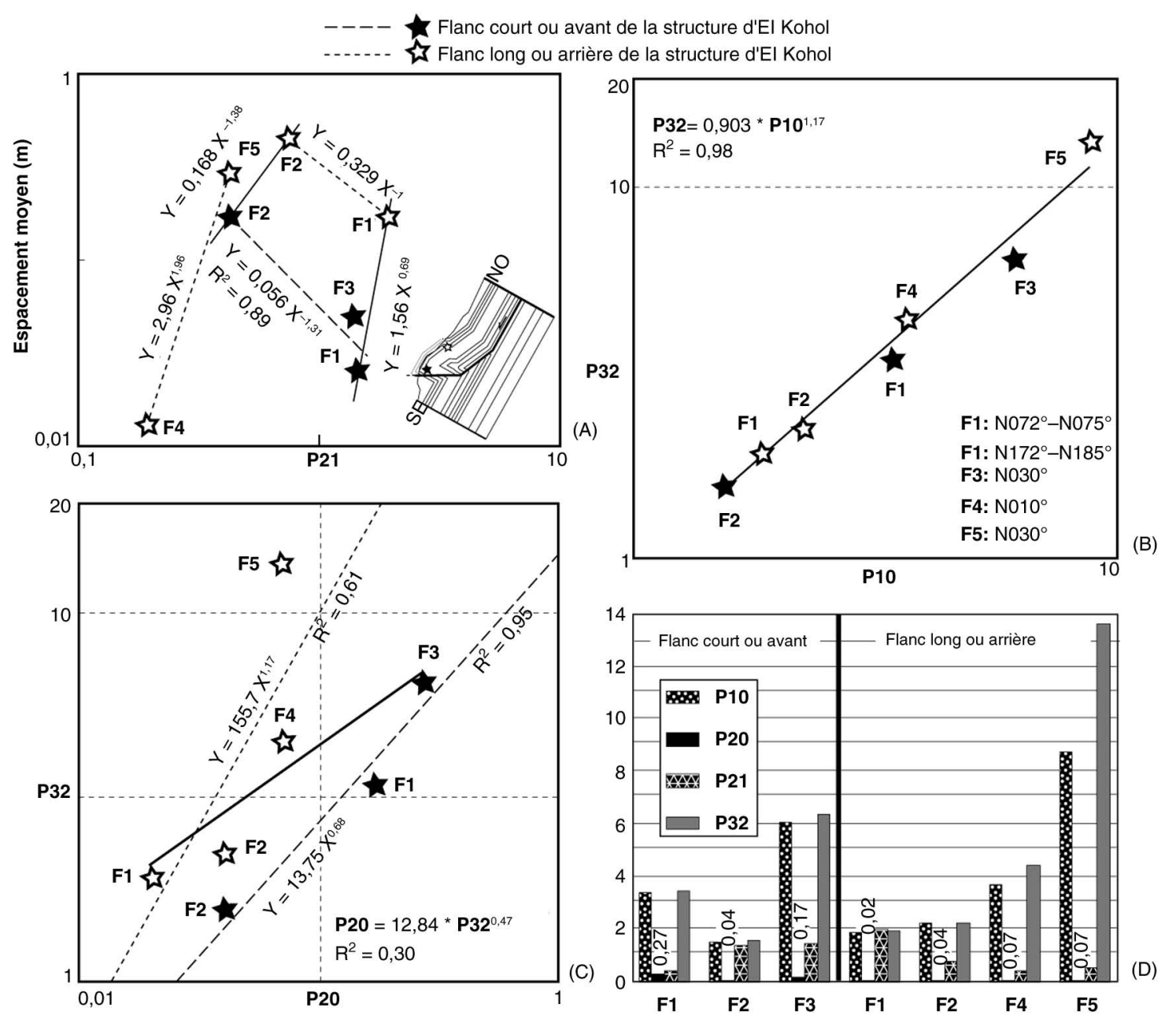

Fig. 5.-(A) Graphe bi-logarithmique des espacements moyens des fractures $(m)$ en fonction du paramètre P21. (B) Graphe bi-logarithmique du paramètre P32 en fonction du paramètre P10. (B) Graphe bi-logarithmique du paramètre P32 en fonction du paramètre P20. (D) Distribution des paramètres dans la structure d'El Kohol.

adopté la méthode décrite par Underwood et al. (2003), qui consiste à digitaliser les fractures et les couches à partir d'une photographie (Bertotti et al., 2007). L'analyse mécanostratigraphique (Fig. 6A) montre une subdivision des trois formations en 12 unités mécaniques. De cette analyse, on distingue de bas en haut une unité mécanique pour la formation d'El Rhelida, sept unités mécaniques pour la formation de M'daouer et 4 unités mécaniques pour la formation de Rhoundjaïa. Le coefficient de variation calculé pour chaque unité montre des valeurs inférieures à 1 , ce qui permet de souligner le contrôle de la fracturation par l'épaisseur des bancs (stratabound). Il est communément admis que l'intensité de la fracturation dépend de l'épaisseur des bancs ou des unités mécaniques (Wu \& Pollard, 1995) et les bancs les plus minces ont tendance à être plus fracturés que les bancs épais (Hobbs, 1967). En effet, la figure 6B illustre bien cette relation où l'intensité de la fracturation est inversement proportionnelle à l'épaisseur de l'unité mécanique (pente négative avec $\mathrm{R}^{2}=0,78$ ). Par ailleurs, un raisonnement mettant en relation l'épaisseur des formations et l'intensité de la fracturation ne permet pas d'arriver à la même conclusion (Fig. 6C). Les valeurs du coefficient d'aplatissement (kurtosis) calculées à partir des espacements des fractures sur les unités mécaniques montrent des valeurs comprises entre $-1,39$ (UmM-5) et 1,03 (UmR-2b). D'après Ruf et al. (1998), les fractures perpendiculaires à la stratification présentent généralement de 


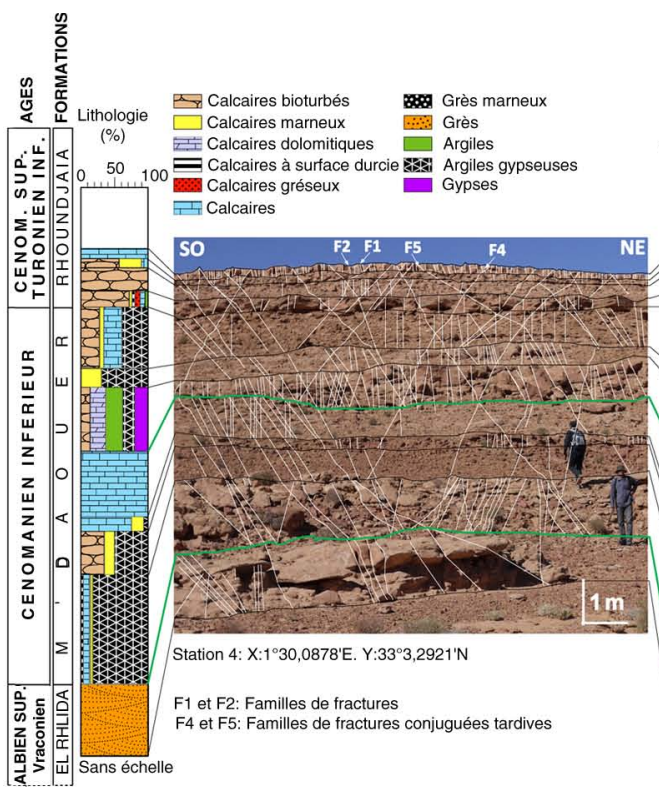

(A)
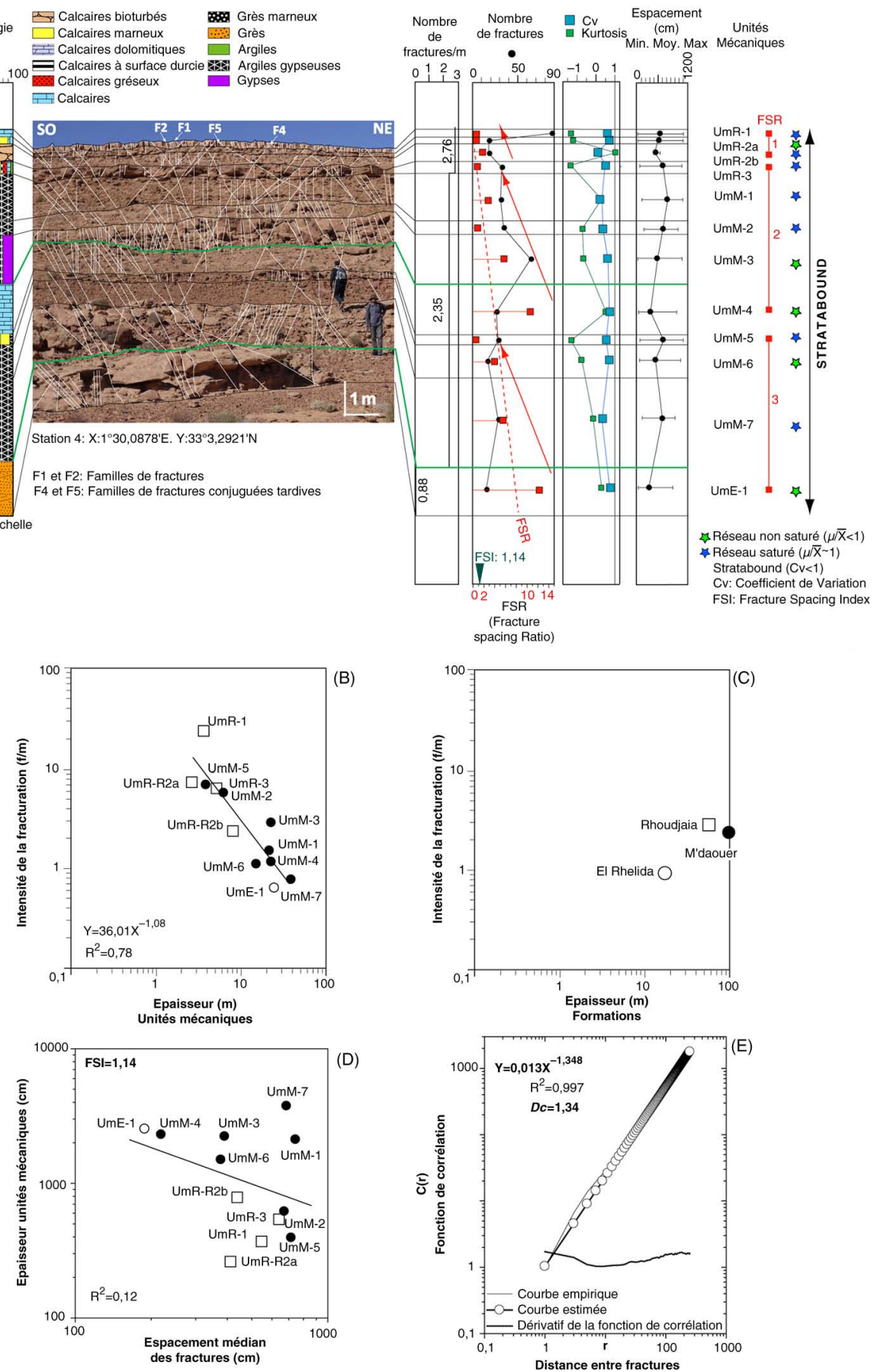

Fig. 6.- Illustration de l'étude sur la mécanostratigraphie des formations observées à El Kohol et analyses géométriques des unités mécaniques (Station 4). (A) De gauche vers la droite: colonne stratigraphique, photographie de l'affleurent avec les fractures, nombre de fractures par mètre par formation; nombre de fractures (P0) intercepté par le profil d'échantillonnage, fracture spacing ratio (FSR) et fracture index $(\mathrm{FI})$; Coefficient de variation $(\mathrm{CV})$ et le Kurtosis; l'espacement moyen $(\mathrm{cm})$ des fractures et les unités mécaniques résultantes. (B) Intensité de la fracturation en fonction de l'épaisseur des unités mécaniques. (C) Intensité de la fracturation en fonction de l'épaisseur des formations. (D) Epaisseur des unités mécaniques en fonction de l'espacement médian des fractures. (E) Résultat de l'analyse fractale du réseau de fractures de la figure 9 par la méthode "The Correlation-Dimension Method". La régression entre les couples de valeurs [Cr, $r$ ] peut être ajustée par une loi de puissance. Le réseau de failles peut être assimilé à un objet fractal de dimension $D c=1,34$. 
faibles valeurs du coefficient d'aplatissement, et leur nombre est fonction de l'épaisseur du banc (stratabound). Notre analyse corrobore ces observations et l'essentiel des résultats est résumé dans le Tableau 3. La relation entre la longueur et l'espacement des fractures peut être quantifiée sous l'appellation de l'index de l'espacement des fractures (FSI pour Fracture Spacing Index) (Narr \& Suppe, 1991; Ruf et al., 1998), qui est égal à la pente de la droite de régression du diagramme mettant en relation l'épaisseur des unités mécaniques en fonction de l'espacement médian des fractures. La courbe tracée (Fig. 6D), peut être ajustée par une loi de puissance dont la pente est égale à 1,14 . Les valeurs typiques pour le FSI sont comprises généralement entre 0,8 et 1,5 (Narr \& Suppe, 1991; Groos, 1993; Engelder et al., 1997), ce qui démontre que la valeur calculé dans notre cas reste dans la fourchette au regard des limites mentionnées dans la littérature. Toutefois, la pente négative soutient que l'espacement le plus faible entre les fractures est observé dans les unités mécaniques les plus épaisses, ce qui est contraire aux résultats publiés dans la littérature (Ladeira \& Priece,1991; Engelder et al., 1997). Selon Eyal et al., (2001), une pente négative indique que l'épaisseur de l'unité mécanique n'est pas seule responsable dans le contrôle des espacements des fractures et une possible explication est à rechercher probablement dans le contraste lithologique. Nous avons calculé pour chaque unité mécanique le coefficient FSR (Fracture Spacing Ratio) défini par Gross (1993) qui est égal à l'épaisseur de l'unité mécanique divisé par l'espacement médian des fractures (Tableau 3). Le FSR permet de montrer qu'au sein d'une même formation, l'intensité de la fracturation peut varier d'une unité mécanique à une autre (Eyal et al., 2001). Le FSR calculé dans notre cas (Tableau 3 et Fig. 6A) montre cette variation avec un gradient moyen décroissant de la base (Formation de Rhelida) vers le sommet (Formation de Rhoundjaïa). Dans le détail, le FSR peut être subdivisé en 3 niveaux (FSR1, FSR2 et FSR3), toutes trois décroissants de la base vers le sommet. Les fortes valeurs matérialisant les bases des niveaux sont liées aux unités mécaniques UmR-2b, UmM-4 et UmE-1 qui correspondent respectivement aux niveaux francs de calcaires bioturbés, calcaires et grès. Cette décroissance pourrait probablement être mise en relation avec le gradient décroissant de

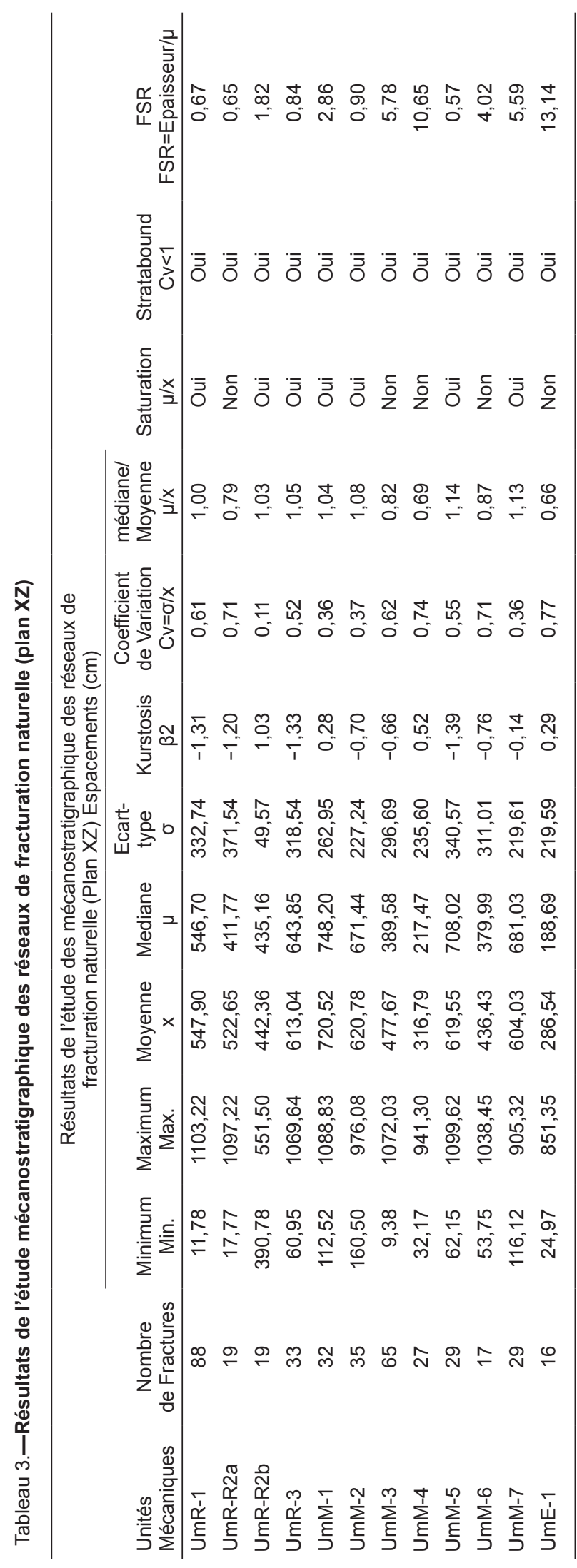


la compaction de la base vers le sommet des formations, donc une diminution probable de la porosité du sommet vers la base. En effet, Engelder et al. (1997), dans leur étude de l'anticlinal de Elk Basin (Montana-Wyoming) relient les valeurs de grande porosité avec les valeurs faibles de FSR. La décroissance du FSR est à mettre aussi avec la diminution de la distance des unités mécaniques par rapport au décro-chevauchement principale d'El Kohol (ADC) (Fig. 1C), sans toutefois écarter l'hypothèse de l'existence de plusieurs événements de déformation ayant aboutit au réseau de fracturation observé actuellement. En plus des familles de fractures F1 et F2, décrites précédemment, l'affleurement montre l'existence de deux familles de fractures F4 et F5, de direction et de pendage respectivement $\mathrm{N} 010^{\circ}-76^{\circ} \mathrm{O}$ et $\mathrm{N} 130^{\circ}-55^{\circ} \mathrm{NE}$ (Fig. 6A). Ces dernières, à l'inverse des autres familles de fractures, présentent une grande extension verticale indépendamment des unités mécaniques qu'elles traversent. L'analyse fractale effectuée sur les fractures digitalisées en figure $6 \mathrm{~A}$ montre un caractère fractal de dimension 2 pour la répartition des fractures. La courbe estimée du graphe bi-logarithmique de la fonction de corrélation $[C(r)]$ en fonction de la distance entre fractures $(r)$ peut être ajustée par une loi de puissance. La dimension fractale $(D c)$ obtenue est de 1,34; donc comprise entre 1 et 2 , avec un coefficient de corrélation $\left(\mathrm{R}^{2}\right)$ de 0,99 (Fig. 6E).

\section{Analyse des bandes de déformation dans la région d'El Kohol}

Les roches poreuses tels le grès, le calcaire, le tuff pyroclastique et la brèche, organisés en réseaux cataclasique présentent parfois des bandes de déformation $(\mathrm{BD})$ en réponse à une déformation par cisaillement, conduisant finalement à des zones de failles géométriquement complexes (Aydin, 1978; Aydin \& Johnson, 1978; Shipton \& Cowie, 2001; Fossen et al., 2007). Les (BD) sont des structures d'épaisseur millimétrique, caractérisés par une réorganisation de grain (glissement de grain par rotation et/ou à de la fracturation associée à de la dilatation, du cisaillement et/ou des mécanismes de compactage). Parmi les différents types de bandes classées en fonction des mécanismes de génèse (cataclastiques, désagrégation, phyllosilicates, solution et cimentation) (Fossen et al., 2007; Schueller et al., 2013), nous nous intéresserons uniquement à un seul type de bandes de déformation présente dans la région. Il s'agit de bandes de déformation compactantes (BDc) (Fig. 7A et 7B) (Mollema \& Antonellini, 1996) et cisaillantes (Fig. 7A) (Du Bernard et al., 2002). Les figures 7A et $7 \mathrm{C}$, illustrent les bandes de déformation observées dans les grès de la formation d'El Rhelida et leurs orientations (Fig. 1B). Ces dernières montrent une augmentation de leurs nombres, ainsi qu'une réduction de leurs espacements à l'approche des accidents. Ainsi, la famille BD1 se concentre à l'approche de l'accident décro-chevauchant d'E1 Kohol (ADC), et les familles $\mathrm{BD} 2 \mathrm{a}$ et $\mathrm{BD} 2 \mathrm{~b}$ sont à mettre en relation avec les décrochements NO-SE dextres (FEK) et N-S senestres (Fig. 1B). L'analyse fractale effectuée par la méthode "The Two Point Correlation Function Method" montre un caractère fractal de dimension 2 pour la répartition des bandes de déformation. $\mathrm{La}$ courbe estimée du graphe bi-logarithmique de la fonction de corrélation $[C(r)]$ en fonction de la distance entre fractures $(r)$ peut être ajustée par une loi de puissance. La dimension fractale $(D c)$ obtenue est de $1,57\left(\mathrm{R}^{2}=0,99\right)$ pour les bandes de déformation (BD1 et BD2a) et de $1,67\left(\mathrm{R}^{2}=0,99\right)$ pour les bandes de déformation (BD1+BD2a+BD2b) (Fig. 7E et $7 \mathrm{~F}$ ). Le graphe mettant en relation les longueurs des bandes de déformation en fonction de l'orientation de ces dernières (Fig. 7G), montre que les bandes de déformation les plus longues dont les orientations sont comprises entre $130^{\circ}$ et $150^{\circ}$, sont liées probablement aux décrochements NO-SE dextres (FEK). La distribution des fréquences cumulées des longueurs de bande déformation montre une loi de puissance entre $272 \mathrm{~mm}$ et $542 \mathrm{~mm}$ (Fig. 7H) caractérisée par une droite de pente $-0,05\left(\mathrm{R}^{2}=0,97\right)$. Notons que la famille F1 est parallèle à la bande de déformation BD1 et au déco-chevauchement principal d'El Kohol et les bandes de déformation BD2a et $\mathrm{BD} 2 \mathrm{~b}$ sont respectivement plus ou moins parallèles aux familles F5 et F2+F4.

\section{Modélisation du réseau de fractures discrètes (DFN)}

A partir des données de terrain (Tableau 2, 3 et 4), nous avons tenté une simulation stochastique du réseau de fracturation par l'approche de la modélisation des réseaux de fractures discrètes (DFN) pour l'unité mécanique UmR-1 (Fig. 6A). Cette méthode 

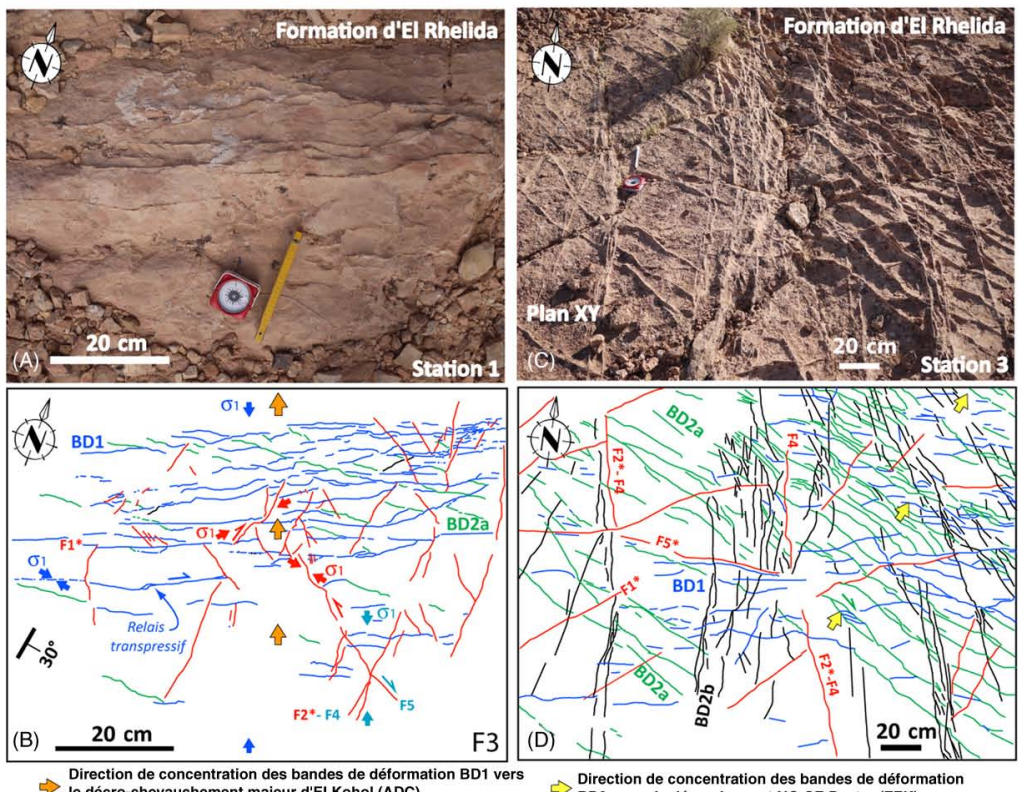

$\Rightarrow$ Direction de concentration des bandes de déformaton

$\Rightarrow$ Direction de concentration des bandes de déformation BD1, BD2a-b: Bandes de déformation; F1, F2 , F3, F4 et F5: Fractures; (") Réactivation des fractures existentes
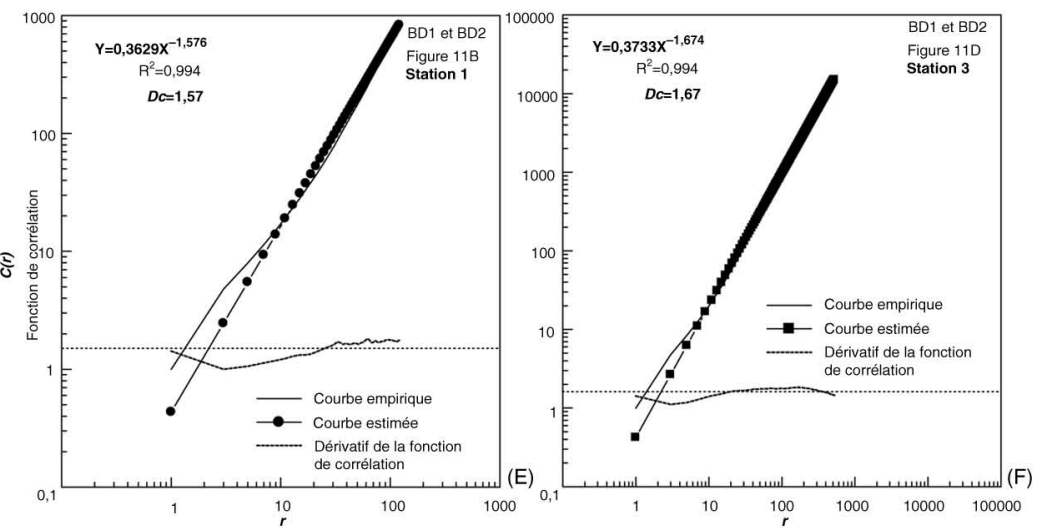

Distance entre bandes $($ pixels $)(1$ pixel $=2,18 \mathrm{~cm})$

Distance entre bandes (pixels) $(1$ pixe $=0,75 \mathrm{~cm})$
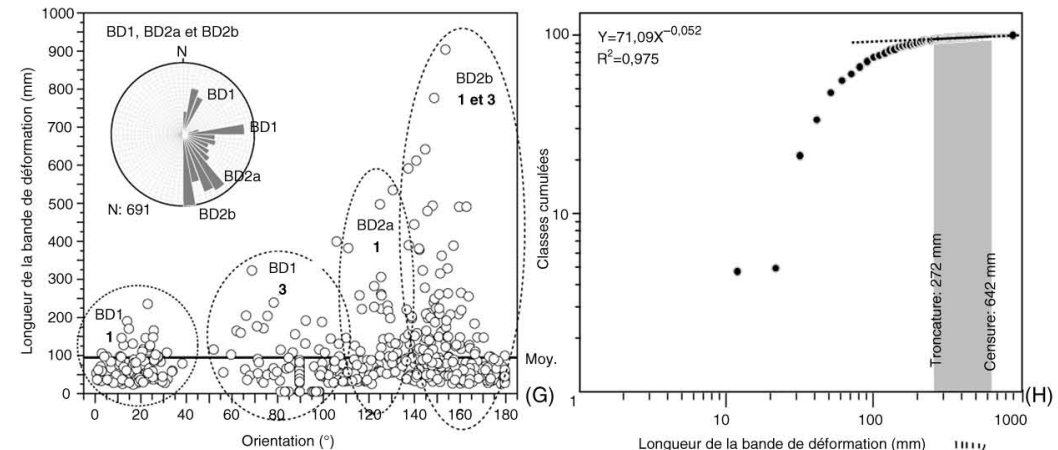

Fig. 7.-Exemples et analyses de bandes de déformation cataclastique dans les grès de la formation d'El Rhelida. (A et B) On peut identifier, une famille de bandes de déformation (BD1), compactantes et cisaillantes, recoupées tardivement par une famille de fractures conjuguées. ( $C$ et $\mathrm{D}$ ) En plus de la famille BD1, on observe 2 familles de bandes déformations (BD2a et BD2b) conjuguées donnant une géométrie anastomosée, recoupés par des fractures. A noter la concentration des bandes en direction des accidents et la réactivation des fractures. ( $E$ et $F$ ) Résultat de l'analyse fractale du réseau de bandes de déformation de la figure 11, par la méthode "The Two Point Correlation Function Method". La régression entre les couples de valeurs [ $\mathrm{Cr}$, r] peut être ajustée par une loi de puissance. Le réseau peut être assimilé à un objet fractal de dimension $D c$ avec $1 \leq D c \leq 2$. (G) Diagramme des longueurs des bandes de deformation en fonction de leurs orientations. (H) Diagramme bi-logarithmique de la distribution des fréquences cumulées des longueurs des bandes de déformation. 
Tableau 4.-Paramètres utilisés pour la modélisation de réseaux de fractures discrètes (DFN) de l'unité mécanique UmR-1, aux niveaux du flanc court et du flanc long de la structure d'El Kohol

\begin{tabular}{|c|c|c|c|c|c|c|c|c|c|}
\hline \multicolumn{10}{|c|}{ Flanc Court ou Avant (Unité UMR-1) } \\
\hline \multirow[b]{2}{*}{ Familles } & \multirow[b]{2}{*}{ Attitude } & \multirow{2}{*}{$\begin{array}{l}\text { Hauteur } \\
(\mathrm{m})\end{array}$} & \multicolumn{3}{|c|}{ Longueur $(\mathrm{m})$} & \multicolumn{3}{|c|}{ Espacement $(\mathrm{m})$} & \multirow[b]{2}{*}{ Distribution } \\
\hline & & & Moyenne & Ecart-type & Variance & Moyenne & Ecart-type & Variance & \\
\hline F1 & $\mathrm{N} 075^{\circ}-84^{\circ} \mathrm{NO}$ & 10,87 & 14,68731214 & 2,549631792 & 6,500622276 & 0,0254 & 0,0108 & 0,0001166 & Log-normale \\
\hline $\mathrm{F} 2$ & $\mathrm{~N} 185^{\circ}-90^{\circ}$ & 10,87 & 5,88203999 & $2,70858 E-15$ & $7,33641 \mathrm{E}-30$ & 0,1683 & 0,14 & 0,0196 & Log-normale \\
\hline F3 & $\mathrm{N} 030^{\circ}-24^{\circ} \mathrm{NE}$ & 10,87 & 5,696805171 & 0,839445287 & 0,704668389 & 0,0506 & 0,0483 & 0,0023329 & Log-normale \\
\hline \multicolumn{10}{|c|}{ Flanc Long ou Arrière (Unité UMR-1) } \\
\hline \multirow[b]{2}{*}{ Familles } & \multirow[b]{2}{*}{ Attitude } & \multirow{2}{*}{$\begin{array}{l}\text { Hauteur } \\
\text { (m) }\end{array}$} & \multicolumn{3}{|c|}{ Longueur $(\mathrm{m})$} & \multicolumn{3}{|c|}{ Espacement $(\mathrm{m})$} & \multirow[b]{2}{*}{ Distribution } \\
\hline & & & Moyenne & Ecart-type & Variance & Moyenne & Ecart-type & Variance & \\
\hline F1 & $\mathrm{N} 072^{\circ}-90^{\circ}$ & 10,87 & 18,53164104 & 0,780570798 & 0,60929077 & 0,1677 & 0,0845 & 0,0071403 & Log-normale \\
\hline $\mathrm{F} 2$ & $\mathrm{~N} 172^{\circ}-90^{\circ}$ & 10,87 & 5,822086163 & 0,598435512 & 0,358125063 & 0,4444 & 0,2538 & 0,0644144 & Log-normale \\
\hline $\mathrm{F} 4$ & $\mathrm{~N} 010^{\circ}-80^{\circ} \mathrm{O}$ & 10,87 & 6,1493865 & $9,42055 E-16$ & 8,87469E-31 & 0,5790 & 0,4155 & 0,1727109 & Log-normale \\
\hline F5 & $\mathrm{N} 130^{\circ}-50^{\circ} \mathrm{NE}$ & 10,87 & 7,909706904 & 1,936140062 & 3,748638338 & 0,114056 & 0,090999 & 0,0082808 & Log-normale \\
\hline
\end{tabular}

permet de définir des modèles de distribution des paramètres relatifs à la densité, à l'orientation et à la taille des fractures à partir des données de terrain (Henrion, 2011). Le nombre et la répartition spatiale des centres des fractures sont générés stochastiquement par le processus de Poisson en fonction d'un modèle de densité de fractures tandis que les caractéristiques géométriques (dimensions, orientation) de chaque fracture sont générées aléatoirement par le tirage de Monte-Carlo dans leurs lois de distribution respectives (Stoyan et al., 1995). Les résultats obtenus pour le flanc court et le flanc long de la structure sont observables en figure 8 . Le réseau $2 \mathrm{D}$ (Fig. $8 \mathrm{~B}$ et $8 \mathrm{D}$ ) fourni résulte de l'intersection du réseau 3D (Fig. 8A et $8 \mathrm{C}$ ) avec un plan de coupe horizontal représenté par le toit de l'unité mécanique UmR-1.

\section{Résultats et discussion}

Les observations et les mesures effectuées sur le terrain dans la région d'El Kohol ont montrés L'existence d'un réseau de fracturation matérialisé par la présence de cinq familles de fractures (F1 à F5) dont les directions etpendages moyens sont comme suit: F1: $\mathrm{N} 073,5^{\circ} \pm 1,5-87^{\circ} \pm 3 \mathrm{NNO} ; \mathrm{F} 2: \mathrm{N} 178,5 \pm 6,5-90^{\circ} ; \mathrm{F} 3$ :

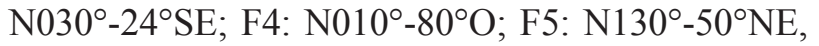
et de trois familles de bandes de déformation BD1, $\mathrm{BD} 2 \mathrm{a}$ et $\mathrm{BD} 2 \mathrm{~b}$ orientées respectivement $\mathrm{E}-\mathrm{O}$ à NE-SO, NO-SE et NNO-SSE à N-S.

Notons que les bandes de déformation montrent une augmentation de leurs nombres, ainsi qu'une réduction de leurs espacements à l'approche des accidents. Ainsi, la famille BD1 se concentre à l'approche de l'accident décro-chevauchant d'El Kohol (ADC), et les familles BD2a et BD2b sont à mettre en relation avec les décrochements NO-SE dextres (FEK) et N-S senestres (Fig. 1B). Antonellini \& Aydin (1995) dans leur étude d'un grès poreux dans les Arches National Parcs (Utah, USA) soulignent que le maximum de densité de bandes de déformation se localise dans la zone de courbure maximale d'un pli. Nos observations ont montré que les bandes de déformation sont localisées et limitées dans l'espace et elles constituent des zones d'endommagement, liées aux accidents comme le suggèrent Shipton \& Cowie (2003) dans leur étude des Grès de Navajo (Utah, USA).

Selon Wang (2005), des relations existent entre les différents paramètres de densité de fractures. Si l'un des paramètres est connu, il peut être estimé en utilisant un autre, en effet Le paramètre P32 en fonction du paramètre $\mathrm{P} 10$ montre une relation proportionnelle (Fig. 5B) et il est en de même pour les paramètres P32 et P20 (Fig. 5C) et l'espacement moyen des fractures $(\mathrm{m})$ et le paramètre P21 (Fig. 5A).

A partir des données de terrain, un réseau de fractures discrètes (DFN) a été établi à l'aide du logiciel FracSim3D, pour le flanc court ou avant et le flanc long ou arrière de la structure. La comparaison des résultats des paramètres Pxy (P20, P21 et $\mathrm{P} 32$ ) obtenus à partir de la modélisation du réseau de fractures discrètes (DFN) avec ceux calculés à partir 


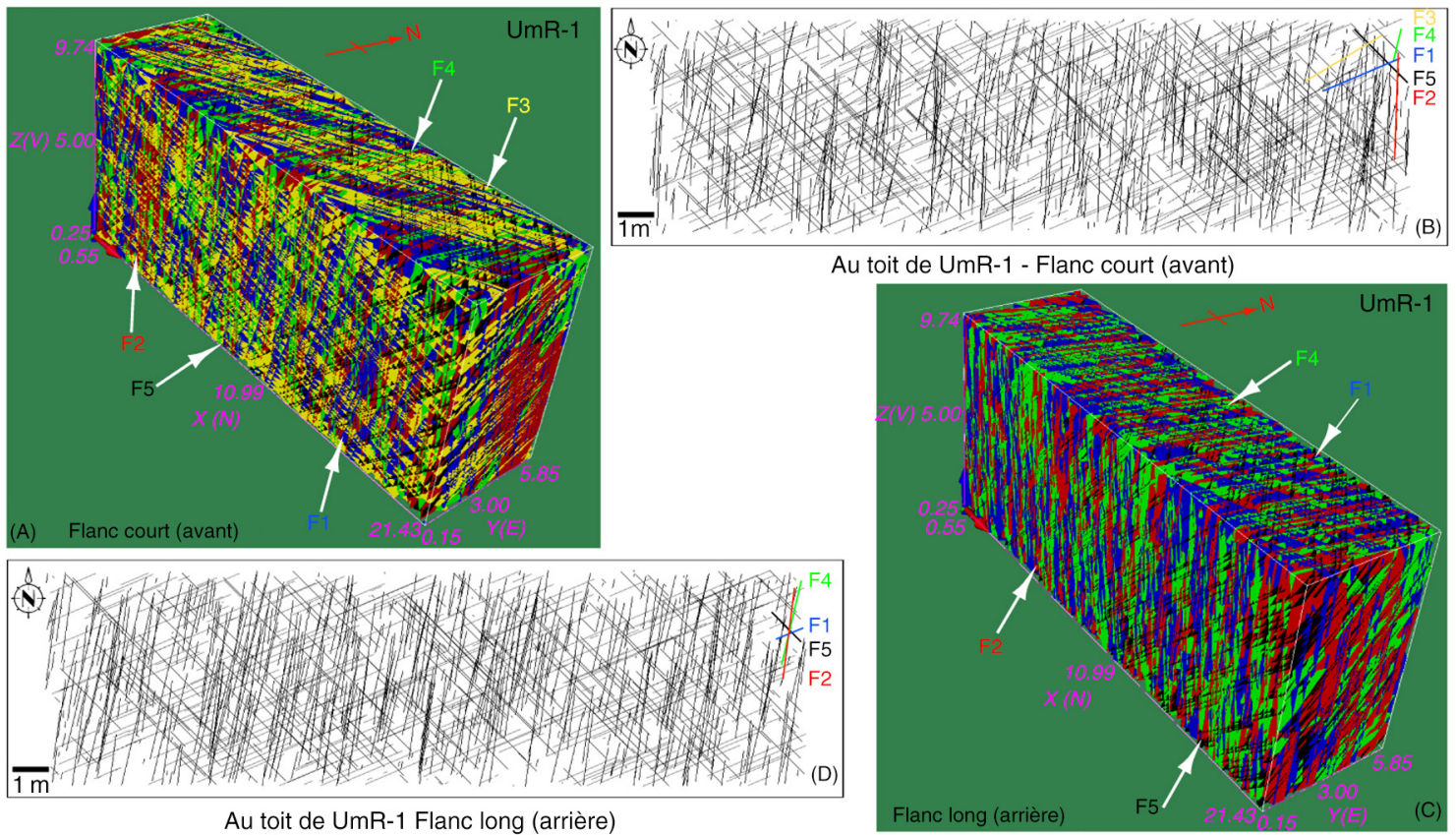

Fig. 8.-Modélisation 3D et 2D de réseaux de fractures discrètes (DFN) dans la structure d'El Kohol de l'unité mécanique UmR-1 (Formation de Rhoundjaïa). (A) Flanc court ou avant. (C) Flanc long ou arrière. (B) Simulation du réseau de fractures discrètes (2D) du flanc court ou avant, au toit de l'unité mécanique UmR-1. (D) Simulation du réseau de fractures discrètes (2D) du flanc long ou arrière au toit de l'unité mécanique UmR-1. (Famille F1 en bleu, famille F2 en rouge, famille F3 en jaune, famille 4 en vert et famille 5 en noir; l'échelle tridimensionnelle est en mètre).

des donnés de terrain montrent de bons coefficients de corrélation $\left(0,50 \leq \mathrm{R}^{2} \leq 0,91\right)$ (Fig. 9A, 9B et $9 \mathrm{C}$ ). Les histogrammes des fréquences des classes de longueurs des fractures toutes familles confondues pour les deux flancs établies à partir des résultats de la modélisation du réseau de fractures discrètes (DFN) montrent des distributions de type négatif exponentiel avec des coefficients de corrélation $\mathrm{R}^{2}$ proches de 1 (Fig. 9D et 9E).

L'analyse fractale effectuée (Fig. 9F) au toit de l'unité mécanique UmR-1 pour les deux flancs de la structure indique un caractère fractal de dimension 2 pour la répartition modélisée (DFN) des fractures. En effet, la courbe estimée du graphe bi-logarithmique de la fonction de corrélation $[C(r)]$ en fonction de la distance entre fractures $(r)$ peut être ajustée par une loi de puissance (Fig. 9F). La dimension fractale (Dc) obtenue est de 1,69; donc comprise entre 1 et 2 , avec un coefficient de corrélation $\left(\mathrm{R}^{2}\right)$ de 0,98 . Les analyses fractales effectuées pour la carte de la région d'El Kohol, et pour les fractures en plan XZ, ainsi que sur les bandes de déformation montrent aussi un caractère fractal de dimension 2 . Les dimensions fractales
(Dc) obtenues sont comprises entre les valeurs 1 et 2 . Les courbes estimées du graphe bi-logarithmique de la fonction de corrélation $[C(r)]$ en fonction de la distance entre fractures $(r)$ peut être ajustée par une loi de puissance avec des coefficients de corrélation très proches de 1. Selon Lasm (2000), les fortes valeurs de la dimension fractale peuvent être mises en relation avec la complexité d'un réseau de fracturation, ce qui signifie l'existence de plus d'une phase de déformation, ce qui corroborent les travaux de Bettahar et al. (1996) dans la région.

Dans leur étude sur les orientations des fractures dans 1'anticlinal de Kuh-e Khaviz (Dezful, Iran). Wennberg et al. (2007) constatent dans leur modèle basé sur celui de Price (1966) que la majorité des fractures présentent un angle fort par rapport au plan de stratification. Ils ont subdivisé les fractures en deux types de familles $\mathrm{T}$ et $\mathrm{R}$ dans le flanc court et le flanc long. Les fractures $\mathrm{T}$ correspondent à une famille de fractures perpendiculaires à l'axe du pli, et qu'ils ont appelées T2 dans le flanc court et T4 dans le flanc long. Les T1 et T3 sont parallèles à $1^{\text {'axe du }}$ pli et sont situées respectivement dans le flanc court 

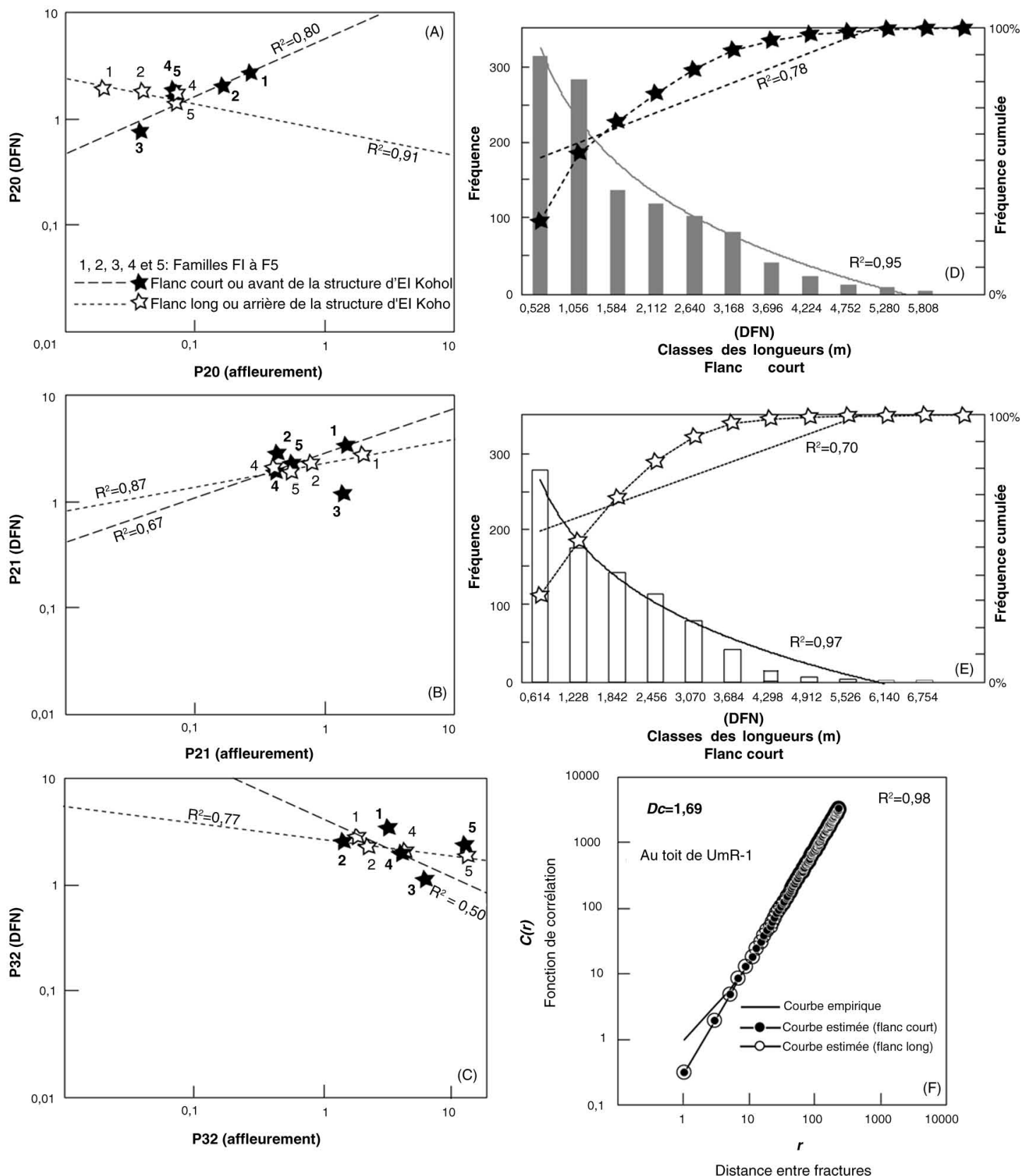

Fig. 9.-Résultats de la modélisation de réseaux de fractures discrètes (DFN). ( $A, B$ et $C$ ) Corrélations des paramètres Pxy (affleurements) avec ceux issus de la modélisation. (D et $E$ ) Histogrammes des fréquences en fonction des longueurs des fractures et loi de distribution (flancs cout et long de la structure, modèle de DFN). (F) Résultat de l'analyse fractale du réseau de fractures déduite de la modélisation (DFN) au toit de l'unité mécanique Um-R1. La régression entre les couples de valeurs [Cr, $r$ ] peut être ajustée par une loi de puissance. Le réseau de fractures peut être assimilé à un objet fractal de dimension $D c=1,69$.

et le flanc long de la structure. L'orientation et la cinématique des fractures $T$ suggèrent qu'elles sont des veines créées en tension et sans cisaillement. D'après Wennberg et al. (2007), ces familles de fractures seraient plus fréquentes dans le flanc long que dans le flanc court. Les familles de fractures $\mathrm{R}$ apparaissent comme des familles conjuguées orientées symétriquement autour des T1 et T2. Ils sont appelées R1 et R2 dans le flanc court, et R3 et R4 dans le flanc long. Le fait que les fractures 


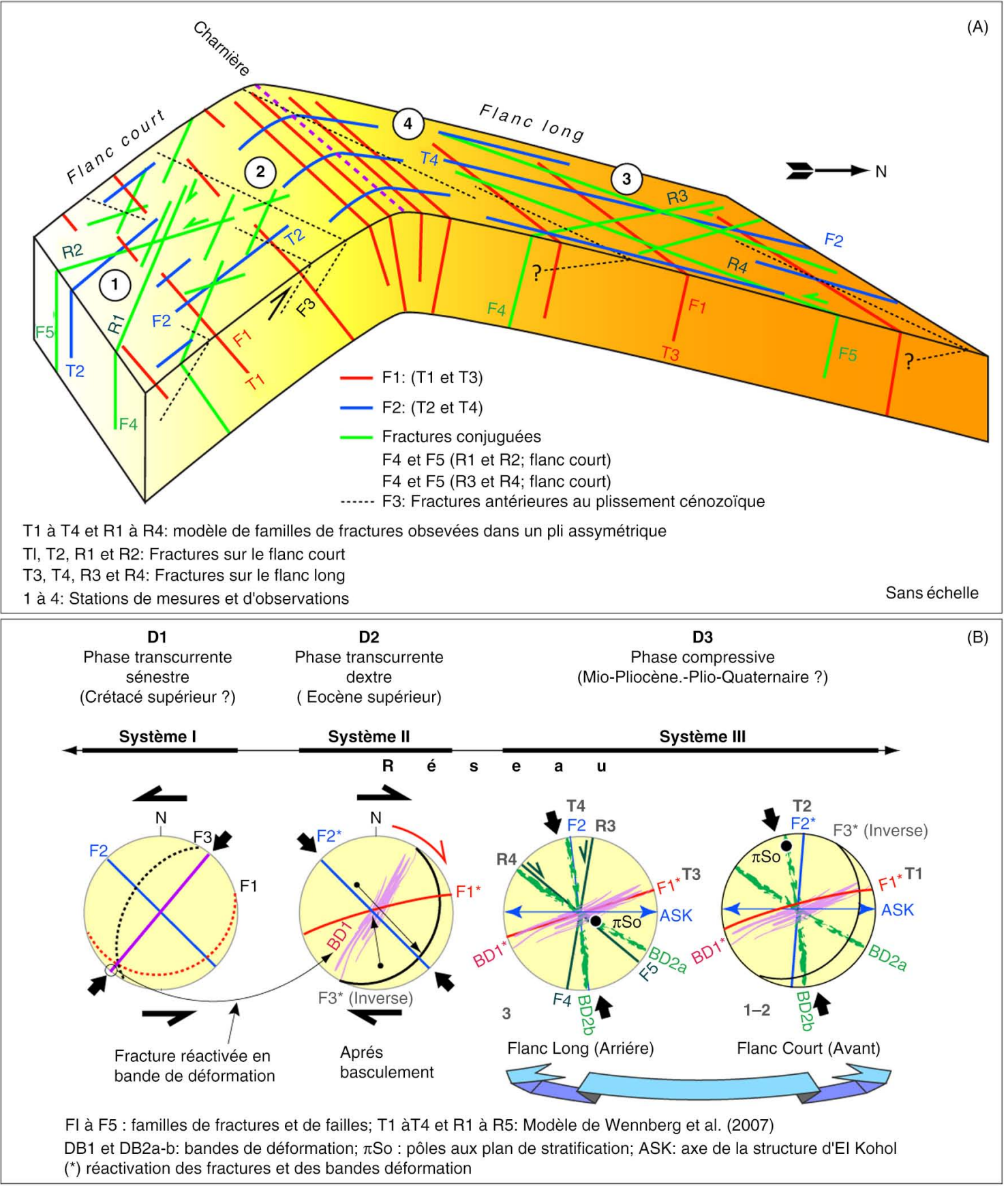

Fig. 10.-Modèle de fracturation naturelle établie pour la structure d'El Kohol (A) et essai de reconstitution chronologique du réseau de fracturation et des bandes de déformation au niveau de la structure d'El Kohol (Canevas iso-angle; hémisphère inférieur) (B).

$\mathrm{R}$ soient des fractures conjuguées suggèrent qu'elles soient ouvertes en cisaillement; mais sur le terrain d'étude, 1 'effet de ce cisaillement n'apparait pas beaucoup. De façon intéressante, la même observation a été effectuée au niveau du pli asymétrique d'El Kohol et la figure 10A montre la concordance entre les familles de fractures mesurées dans notre étude et le modèle de Wennberg et al. (2007). Dans leurs études des réseaux de fracturation des structures de Bina Bawi et de Taq Taq dans les monts Zagros (Kurdistan), Awdal et al. (2013) arrivent aux mêmes constatations. De façon intéressante, la 
même observation a été effectuée au niveau du pli asymétrique de Sheep Mountain (Wyoming, USA) (Bellahsen et al., 2006).

Dans le flanc sud (court ou avant) de la structure, les fractures de la famille F3, plus anciennes sont réactivées en failles inverses durant le basculement et portent les bandes de déformation DB1. Selon Amrouch (2010). La réactivation des fractures préexistantes agit essentiellement sur les contraintes à la fois par un relâchement de ces dernières et par une réorientation de leurs directions, cette variation de l'état de contrainte peut avoir comme effet d'inhiber ou favoriser la formation de certaines familles de fractures.

L'analyse mécanostratigraphique réalisée montre une subdivision des trois formations en 12 unités mécaniques. On distingue de bas en haut une unité mécanique pour la formation d'El Rhelida, quoi incomplète, sept unités mécaniques pour la formation de M'daouer et 4 unités mécaniques pour la formation de Rhoundjaïa, avec le contrôle de la fracturation par l'épaisseur (stratabound). L'épaisseur de l'unité mécanique n'est pas seule responsable du contrôle des espacements des fractures et une possible explication est à rechercher probablement dans le contraste lithologique, le phénomène de compaction et la position structurale. Nous avons essayé d'établir une reconstitution chronologique de la genèse du réseau de fracturation et des bandes de déformation au niveau de la structure d'El Kohol (Fig. 10B). Le modèle ainsi obtenu pour cette étude reste cohérent avec les phases de déformation décrites par Bettahar et al. (1996) dans la région.

\section{Conclusion}

Les observations et les mesures effectuées ont montrés l'existence de cinq familles de fractures et de trois familles de bandes de déformation. Le flanc long ou arrière de la structure présente un réseau ou les fractures sont plus longues et moins denses. A l'inverse, le flanc court ou avant présente les fractures les moins longues et les plus denses.

Le modèle de distribution des espacements des fractures obéit à la loi de puissance et il est cohérent statistiquement de l'échelle de l'affleurement à celle de la carte et il en est de même pour le modèle de distribution des longueurs.
L'analyse des espacements entre fractures montre pour chaque famille de fractures une distribution de type log-normale en accord avec un modèle dit aléatoire. Les courbes de distribution des fréquences cumulées des espacements entre fractures pour chaque famille peuvent être ajustées par une loi de puissance avec des coefficients de corrélation très proches de 1. L'épaisseur de l'unité mécanique n'est pas seule responsable du contrôle des espacements des fractures.

Les bandes de déformation observées montrent une augmentation de leurs nombres et une réduction de leurs espacements à l'approche des accidents majeurs.

Les analyses fractales effectuées sur les failles de la région d'El Kohol, et pour les fractures, ainsi que sur les bandes de déformation montrent un caractère fractal de dimension 2.

La comparaison des résultats des paramètres Pxy obtenus à partir de la modélisation du réseau de fractures discrètes (DFN) avec ceux calculés à partir des donnés de terrain montrent de bons coefficients de corrélation. Contrairement au flanc long qui présente un réseau ou les fractures sont moins denses et plus longues, le flanc court présente quant à lui, un réseau de fractures plus dense et moins longues.

\section{REMERCIEMENTS}

Les auteurs remercient vivement $\mathrm{T}$. Zouaghi et le rapporteur anonyme pour leurs remarques et commentaires. Nous tenons aussi, à remercier la Division Technologies et Développement de Sonatrach pour le soutien logistique sur le terrain. Mr. Chaoshui Xu (Université d'Adelaïde, Australie) pour avoir mis à notre disposition le logiciel FracSim 3D. La présente note est le résultat des travaux entrepris dans l'Atlas saharien dans le cadre d'un projet entre Sonatrach et l'Université Abou Bekr Belkaïd-Tlemcen ayant pour titre: Les événements anoxiques dans les bassins nord-algériens: sédimentologie, biostratigraphie et implication pétrolière (contrat $\left.\mathrm{N}^{\circ} 01 / 2013\right)$.

\section{Références}

Allègre, J.C.; Lemouel, J.L. \& Provost, A. (1982). Scaling rules in rock fracture and possible implications for earthquake prediction. Nature, 297: 47-49. http:// dx.doi.org/10.1038/297047a0

Allmendinger, R.W. (2013). Logiciel FaultKin 6.1.0. Cornell University, U.S.A. http://www.geo.cornell. edu/geology/faculty/RWA/programs/stereonet.html

Allmendinger, R.W.; Cardozo, N. \& Fisher, D. (2012). Structural geology algorithms: Vectors and tensors. Cambridge University Press, 302 pp. 
Amrouch, K. (2010). Apport de l'analyse microstructurale à la compréhension des mécanismes de plissement: Exemples de structures plissées aux USA (Wyoming) et en Iran (Zagros). Thèse de Doctorat, Université Pierre et Marie Curie, Paris 6, France, 479 pp.

Antonellini, M. \& Aydin, A. (1995). Effect of faulting on fluid flow in porous sandstones: geometry and spatial distribution. AAPG Bulletin, 79: 642-671.

Awdal. A.H.; Braathen, A.; Wennberg, O.P. \& Sherwani, G.H. (2013). The characteristics of fracture networks in the Shiranish Formation of the Bina Bawi Anticline; comparison with the Taq Taq Field, Zagros, Kurdistan, NE Iraq. Petroleum Geoscience, 19: 139-155. http://dx.doi.org/10.1144/petgeo2012-036

Aydin, A. (1978). Small faults formed as deformation bands in sandstone. Pure and Applied Geophysics, 116: 913-930. http://dx.doi.org/10.1007/BF00876546

Aydin, A. \& Johnson, A.M. (1978). Development of faults as zones of deformation bands and as slip surfaces in sandstone. Pure Applied Geophysics, 116: 931-942. http://dx.doi.org/10.1007/BF00876547

Bekkers, R.W.G. (2013). Discrete Fracture Networks in carbonate anticlines: an example from the Berda Anticline in Southern Tunisia. PhD thesis, Delft University of Technology, The Netherlands, $96 \mathrm{pp}$.

Bellahsen, N.; Fiore, P. \& Pollard, D.D. (2006). The role of fractures in the structural interpretation of Sheep Mountain Anticline, Wyoming. Journal of Structural Geology, 28 (5): 850-867. http://dx.doi.org/10.1016/j. jsg.2006.01.013

Bettahar, A.; Habani, H. \& Medaouri, M. (1996). Analyse structural d'un tronçon de l'ASA. El Kohol, Brézina. Atlas Saharien occidental. Bulletin du Service Géologique de l'Algérie, 8 (1): 81-94.

Bisdom, K. (2011). Modelling 3D Discrete Fracture Networks using 2D outcrop data. Master Thesis, Delft University of Technology, The Netherlands, $166 \mathrm{pp}$.

Bodin, J. \& Razack, M. (1999). L'analyse d'images appliquée au traitement automatique de champs de fractures. Propriétés géométriques et lois d'échelles. Bulletin de la Société Géologique de France, 170: 579-593.

Bonnet, E.; Bour, O.; Odling, N.E.; Davy, P.; Main, I.G.; Cowie, P.A. \& Berkowitz, B. (2001). Scaling of fracture systems in geological media. Reviews of Geophysics, 39: 347-383. http://dx.doi.org/10.1029/ 1999RG000074

Bour, O. \& Davy, P. (1999). Clustering and size distribution of fault patterns: Theory and measurements. Geophysical Research Letters, 26: 2001-2004. http:// dx.doi.org/10.1029/1999GL900419

Bertotti, G.; Hardebol, J.K.; Koppen, T.-V. \& Luthi, M. (2007). Toward a quantitative definition of mechanical units: New techniques and results from an outcropping deep-water turbidite succession (Tanqua-Karoo Basin, South Africa). AAPG Bulletin, 91: 1085-1098. http:// dx.doi.org/10.1306/03060706074
Bracène, R.; Bellahcène, A.; Bekkouche, D.; Mercier, E. \& Frizon de Lamotte, D. (1999). The thin-skinned style of the South Atlas Front in central Algeria. L'explorateur, 13: 15-22.

Chilès, J.P. (1988). Fractal and geostatistical methods for Modeling of a fracture network. Mathematical Geology, 20 (6): 631-653. http://dx.doi.org/10.1007/ BF00890581

Davy, P.; Sornette, A. \& Sornette, D. (1990). Some consequences of a proposed fractal nature of continental faulting. Nature, 348: 56-58. http://dx.doi. org/10.1038/348056a0

Deleze, J.Y.; Jaboyedoff, M. \& Rouiller, J.D. (2005). Logiciel MaterCliff 1.0. Centre de recherche sur l'environnement alpin, Sion, Suisse.

Dershowitz, W.S. \& Einstein, H.H. (1988). Characterizing rock joint geometry with joint. Rock Mechanics and Rock Engineering, 21: 21-51. http://dx.doi.org/ 10.1007/BF01019674

Dershowitz, W.S. \& Herda, H.H. (1992). Interpretation of fracture spacing and intensity. In: Proceedings of the 33rd U.S. Symposium on Rock Mechanics (Tillerson, J.R. \& Wawersik, W.R., Eds.), Rotterdam, Balkema, Netherlands, 757-766.

Du Bernard, X.; Eichhubl, P. \& Aydin, A. (2002). Dilation bands: A new form of localized failure in granular media. Geophysical Research Letters, 29: 1-4. http:// dx.doi.org/10.1029/2002GL015966

Engelder, T.; Gross, M.R. \& Pinkerton, P. (1997). Joint development in clastic rocks of the Elk Basin anticline, Montana-Wyoming. In: Fractured Reservoirs: Characterization and Modeling (Hoak, T.; Klawitter, A. \& Blomquist. P., Eds.), Rocky Mountain Association of Geologists Guidebook, Denver, Colorado, 1-18.

Eyal, Y.; Gross, M.; Engelder, T. \& Becker, A. (2001). Joint development during fluctuation of regional stress field in southern Israel. Journal of Structural Geology, 23: 279-296. http://dx.doi.org/10.1016/ S0191-8141(00)00096-1

Falconer, K. (1990). Fractal Geometry. Wiley and Sons Editions, Chichester, U.K., 400 pp.

Fossen, H.; Schultz, R.A.; Shipton, Z.J. \& Mair. K. (2007). Deformation bands in sandstone: a review. Journal of the Geological Society, London, 164: 755-769. http://dx.doi.org/10.1144/0016-76492006-036

Frankhauser, P.; Tannier, C. \& Vuidel, G. (2005). Logiciel Fractalyse. 2.3.2. Ministère de l'EquipementUniversité Franche-Comté, France. http://www. fractalyse.org/fr-download.html

Gillespie, P.A.; Howard, C.B.; Walsh, J.J. \& Watterson, J. (1993). Measurement and characterisation of spatial distributions of fractures. Tectonophysics, 226: 113-141. http://dx.doi.org/10.1016/0040-1951(93) 90114-Y

Grassberger, P. \& Procaccia, I. (1983). Characterization of strange attractor. Physical Review Letters, 50: 346-349. http://dx.doi.org/10.1103/PhysRevLett.50.346 
Gross, M.R. (1993). The origin and spacing cross joints: examples from Montery Formation, Santa Barbara coast-line, California. Journal of Structural Geology, 5: 737-751. http://dx.doi.org/10.1016/0191-8141(93) 90059-J

Guemache, M.A.; Addoum, B.; Machane, D.; Gharbi, S.; Ymmel, H.; Djadia, L. \& Djellit, H. (2011). Apport de la morphotectonique et des profils sismique reflexion 2D dans l'analyse du front Sud de l'Atlas saharien occidental: exemple du pli d'El Kohol (région de Laghouat, Algérie). Mémoires du Service Géologique de l'Algerie, 17: 107-123.

Guillemot, J. \& Estorges, P. (1981). Notice de la carte de Brézina au 1/200000. Direction de la Géologie, Direction des Mines et de la Géologie, Ministère de l'Industrie Lourde, Algérie. 45 pp.

Habani, H. \& Haddoum, H. (2006). Étude comparative du comportement cinématique de l'accident Sud Atlasique: exemples de la région d'El Kohol et de l'axe chaîne des Guerguitts-Monts de Ferkane (Atlas Saharien Algérien). Bulletin du Service Géologique National, 17 (1): 29-45.

Hentschel, H.G.E. \& Procaccia, I. (1983). The infinite number of generalized dimensions of fractals and strange attractors. Physica D, 8: 435-444. http:// dx.doi.org/10.1016/0167-2789(83)90235-X

Henrion, V. (2011). Approche pseudogénétique pour la simulation stochastique de la géométrie $3 \mathrm{D}$ de réseaux de fractures et karstiques. Thèse de Doctorat en Géosciences, 1'Institut National Polytechnique de Lorraine, France, 173 pp.

Hobbs, D.W. (1967). The formation of tension joints in sedimentary rocks: an explanation. Geological Magazine, 104: 550-556. http://dx.doi.org/10.1017/ S0016756800050226

Kruhl, J.H. (2013). Fractal-geometry techniques in the quantification of complex rock structures: a special view on scaling regimes, inhomogeneity and anisotropy. Journal of Structural Geology, 46: 2-21. http://dx.doi. org/10.1016/j.jsg.2012.10.002

Ladeira, F.L. \& Price, N.J. (1981). Relationship between fracture spacing and bed thickness. Journal of Structural Geology, 3: 179-183. http://dx.doi.org/10.1016/ 0191-8141(81)90013-4

Lasm, T. (2000). Hydrogéologie des réservoirs fracturés de socle: Analyses statistique et géostatistique de la fracturation et des propriétés hydrauliques. Application à la Région des Montagnes de Côte d'Ivoire (Domaine Archéen). Thèse de doctorat, Université de Poitiers, France, 274 pp.

Laubach, S.E.; Olson, J.E. \& Gross, M.R. (2009). Mechanical and fracture stratigraphy. AAPG Bulletin, 93: 1413-1426. http://dx.doi.org/10.1306/07270909094

Lavenu, A.P.C.; Lamarche, J.; Galllois, A. \& Gauthier, B.D.M. (2013). Tectonic versus diagenetic origin of fractures in a naturally fractured carbonate reservoir analog (Nerthe anticline, southeastern France).
AAPG Bulletin, 97: 2207-2232. http://dx.doi.org/ 10.1306/04041312225

Macé, L. (2008). Caractérisation et modélisation numériques tridimensionnelles des réseaux de fractures naturelles- Application au cas des réservoirs. Thèse de Doctorat, Institut National Polytechnique de Lorraine, France, $172 \mathrm{pp}$.

Mandelbrot, B. (1975). Les objets fractals. Editions Flammarion, Paris, France, 212 pp.

Marok, A. (1996). Stratigraphie, sédimentologie et interprétations géodynamiques du Lias-Début Dogger: exemple de sédimentation carbonatée de plate-forme en Oranie (Monts de Sidi El Abed, Hautes Plaines, Algérie occidentale). Documents des Laboratoires de Géologie Lyon, 141, 199 pp.

Marrett, R.A. \& Allmendinger, R.W. (1990). Kinematic analysis of fault-slip data. Journal of Structural Geology, 12: 973-986. http://dx.doi.org/10.1016/ 0191-8141(90)90093-E

Mollema, P.N. \& Antonellini, M.A. (1996). Compaction bands: A structural analog for anti-mode I cracks in aeolian sandstone. Tectonophysics, 267: 209-228. http://dx.doi.org/10.1016/S0040-1951(96)00098-4

Mustapha, H. (2005). Simulation numérique de l'écoulement dans des milieux fracturés tridimensionnels. Thèse de Doctorat, Université de Rennes, France, $151 \mathrm{pp}$.

Narr, W. (1996). Estimating average fracture spacing in subsurface rock. AAPG Bulletin, 80: 1565-1586.

Narr, W. \& Suppe, J. (1991). Joint spacing in sedimenlary rocks. Journal of Structural Geology, 11: 1037-1048. http://dx.doi.org/10.1016/0191-8141(91)90055-N

National Research Council. Committee on Fracture Characterization and Fluid Flow (1996). Rock Fractures and Fluid Flow: Contemporary Understanding and Applications. National Academy Press, Washington D.C., $551 \mathrm{pp}$.

Nelson, R.A. (2001). Geologic Analysis of Naturally Fractured Reservoirs. Gulf Professional Publishing Editions, Houston, 350 pp.

Odling, N.E.; Gillespie, P.; Bourgine, B.; Castaing, C.; Chilès, J.P.; Christensen, N.P.; Fillion, E., Genter, A.; Olsen, C.; Thrane, L.; Trice, R.; Aarseth, E.; Walsh, J.J. \& Watterson, J. (1999). Variations in fracture system geometry and their implications for fluid flow in fractured hydrocarbon reservoirs. Petroleum Geosciences, 5: 373-384. http://dx.doi.org/10.1144/ petgeo.5.4.373

Ortega, J.O.; Marett, R.A. \& Laubach, S.E. (2006). A scale-independent approach to fracture intensity and average spacing measurement. AAPG Bulletin, 90: 193-208. http://dx.doi.org/10.1306/08250505059

Peacock, D.C.P. (2001). The temporal relationship between joints and faults. Journal of Structural Geology, 23: 329-341. http://dx.doi.org/10.1016/ S0191-8141(00)00099-7

Peacock, D.C.P. \& Mann, A. (2005). Evaluation of the controls on fracturing in reservoir rocks. Journal of 
Petroleum Geology, 28: 385-396. http://dx.doi.org/ 10.1111/j.1747-5457.2005.tb00089.x

Pollard, D.D. \& Segall, P. (1987). Theoretical displacements and stresses near fractures in rock: with applications to faults, joint, veins, dikes and solution surfaces. In: Fracture mechanics of rock (Atkinson, B.K., Ed.), Academic Press, San Diego, 71-110.

Price, N.J. (1966). Fault and Joint Development in Brittle and Semi-brittle Rocks. Pergamon, Oxford, $176 \mathrm{pp}$.

Priest, S.D. \& Hudson, J.A. (1976). Discontinuity spacings in rock. International Journal of Rock Mechanics and Mining Sciences \& Geomechanics Abstracts, 13: 135-148. http://dx.doi.org/10.1016/0148-9062 (76)90818-4

Rerbal, L. (2008). Le Crétacé Supérieur du Djebel El Kohol (Le passage Cénomanien-Turonien dans la région d'El Kohol, Atlas saharien, Algérie). Mémoire de Magistère, Université Abou Bekr Belkaïd, Tlemcen, Algérie, $63 \mathrm{pp}$.

Ruf. C.J.; Rust, K.A. \& Engelder, T. (1998). Investigating the effect of mechanical discontinuites on joint spacing. Tectonophysics, 295: 245-257. http://dx.doi.org/ 10.1016/S0040-1951(98)00123-1

Shipton, Z.K. \& Cowie, P.A. (2001). Analysis of threedimensional damage zone development over $\mathrm{m}$ to $\mathrm{km}$ scale range in the high-porosity Navajo sandstone, Utah. Journal of Structural Geology, 23: 1825-1844. http://dx.doi.org/10.1016/S0191-8141(01)00035-9

Shipton, Z.K. \& Cowie, P.A. (2003). A conceptual model for the origin of fault damage zone structures in highporosity sandstone: Journal of Structural Geology, 25: 333-344. http://dx.doi.org/10.1016/S0191-8141(02) 00037-8

Sornette, A.; Davy, P. \& Sornette, D. (1993). Fault growth in brittle-ductile experiments and the mechanics of continental collisions. Journal of Geophysical Research, 98: 111-139. http://dx.doi.org/10.1029/92JB01740

Schueller, S.; Alvar Braathenn, A.; Fossen, H. \& Tveranger, Y. (2013). Spatial distribution of deformation bands in damage zones of extensional faults in porous sandstones: Statistical analysis of field data.
Journal of Structural Geology, 52: 148-162. http:// dx.doi.org/10.1016/j.jsg.2013.03.013

Stoyan, D.; Kendall, W. \& Mecke, J. (1995). Stochastic Geometry and its applications. Wiley \& Sons, New York, 345 pp.

Terzaghi, R.D. (1965). Sources of errors in joint surveys. Géotechnique, 15: 287-304.

Turcotte, D.L. (1986). A fractal model for crustal deformation. Tectonophysics, 132: 261-269. http:// dx.doi.org/10.1016/0040-1951(86)90036-3

Turcotte, D.L. (1992). Fractals and Chaos in Geology and Geophysics. Cambridge University Press, Cambridge, $221 \mathrm{pp}$.

Underwood, C.A.; Cooke, M.L.; Simo, J.A. \& Muldoon, M.A. (2003). Stratigraphic controls on vertical fracture patterns in Silurian dolomites, northeastern Wisconsin. AAPG Bulletin, 87: 121-142. http:// dx.doi.org/10.1306/072902870121

Wang, X. (2005). Stereological Interpretation of Rock Fracture Traces on Borehole Walls and Other Cylindrical Surfaces. PhD Thesis. Faculty of the Virginia Polytechnic Institute and State University, Blacksburg, Virginia, USA, 123 pp.

Wennberg, O.P.; Azizzadeh, M.; Aqrawi, A.A.M.; Blanc, E.; Brockbank, P.; Lyslo, K.B.; Pickard, N.; Salem, L.D. \& Svana, T. (2007). The Khaviz Anticline: an outcrop analogue to giant fractured Asmari Formation reservoirs in SW Iran. The Geological Society of London. Special Publications, 270: 33-42. http:// dx.doi.org/10.1144/GSL.SP.2007.270.01.02

Wu, H. \& Pollard, D.D. (1995). An experimental study of the relationships between joint spacing and layer thickness. Journal of Structural Geology; 17:887-905. http://dx.doi.org/10.1016/0191-8141(94)00099-L

$\mathrm{Xu}$, C. \& Dowd, P. (2010). A new computer code for discrete fracture network modelling. Computers \& Geosciences, 36: 292-301. http://dx.doi.org/10.1016/j. cageo.2009.05.012

Zazoun, R.S. (2008). The Fadnoun area, Tassili-n-Azdjer, Algeria: fracture network geometry analysis. Journal of African Earth Sciences, 50: 273-285. http://dx.doi. org/10.1016/j.jafrearsci.2007.10.001 